\title{
Finite Generation of a Canonical Ring
}

\author{
Yujiro Kawamata
}

\begin{abstract}
The purpose of this note is to review an algebraic proof of the finite generation theorem due to Birkar-Cascini-Hacon-McKernan [5] whose method is based on the Minimal Model Program (MMP). An analytic proof by Siu [57] will be reviewed by Mihai Paun.
\end{abstract}

\section{Introduction}

The finite generation of canonical rings was a problem considered by Zariski [64], and the proof in the case of dimension 2 due to Mumford [41] in the appendix of [64] is one of the motivations towards the minimal model theory of higher dimensional algebraic varieties.

Let $X$ be a smooth projective variety defined over a field $k, D$ a divisor on $X$, and $\mathcal{O}_{X}(D)$ the associated invertible sheaf. Many problems in algebraic geometry are translated into questions on the vector space of holomorphic sections $H^{0}(X, D)=H^{0}\left(X, \mathcal{O}_{X}(D)\right)$. The Riemann-Roch problem is to determine this vector space. For example, the Riemann-Roch theorem tells us that the alternating sum $\sum_{p=0}^{n}(-1)^{p} \operatorname{dim} H^{p}\left(X, \mathcal{O}_{X}(D)\right)$ is expressed in terms of topological invariants.

Instead of considering a single vector space, we look at the graded ring

$$
R(X, D)=\bigoplus_{m=0}^{\infty} H^{0}(X, m D)
$$

called the section ring for the pair $(X, D)$, where we use the additive notation for divisors instead of multiplicative one for sheaves; we have $\mathcal{O}_{X}(m D)=$ $\mathcal{O}_{X}(D)^{\otimes m}$. There are obvious multiplication homomorphisms

$$
H^{0}\left(X, m_{1} D\right) \otimes H^{0}\left(X, m_{2} D\right) \rightarrow H^{0}\left(X,\left(m_{1}+m_{2}\right) D\right)
$$

and the section ring becomes a graded algebra over the base field $k=$ $H^{0}\left(X, \mathcal{O}_{X}\right)$. The following question arises naturally:

QUESTION 1.1. Is the section ring $R(X, D)$ finitely generated as a graded algebra over $k$ ? 
If $D$ is ample, then the answer is yes. On the other hand, if $\operatorname{dim} X=2$, then there exists an example where $R(X, D)$ is not finitely generated $([\mathbf{6 4}])$. But the canonical divisor $K_{X}$, a divisor corresponding to the sheaf of holomorphic $n$-forms $\Omega_{X}^{n}$ for $n=\operatorname{dim} X$, has a special status in algebraic geometry, and the canonical ring $R\left(X, K_{X}\right)$ is finitely generated:

TheOREM 1.2 (Finite Generation Theorem). Let $X$ be a smooth projective variety defined over a field $k$ of characteristic 0 . Then the canonical ring $R\left(X, K_{X}\right)$ is always finitely generated as a graded algebra over $k$.

The canonical divisor, or more precisely its linear equivalence class, is special in many senses:

- It is naturally attached to any smooth variety as the determinant bundle of the cotangent bundle.

- It is related to the Serre-Grothendieck duality.

- The canonical ring is a birational invariant, i.e., if $f: X \rightarrow Y$ is a birational morphism between smooth projective varieties, then the natural homomorphism $f_{*}: R\left(X, K_{X}\right) \rightarrow R\left(Y, K_{Y}\right)$ is an isomorphism.

There are two proofs, an algebraic one using the MMP by Birkar-CasciniHacon-McKernan [5] and an analytic one using complex analysis by Siu [57]. We note that the algebraic proof yields the finite generation for varieties which are not necessarily of general type because the proof is naturally "logarithmic" as we shall explain later.

A geometric implication of the finite generation theorem is the existence of the canonical model Proj $R\left(X, K_{X}\right)$. It is a traditional basic tool in the investigation of algebraic surfaces of general type.

The anti-canonical ring $R\left(X,-K_{X}\right)$ is not necessarily finitely generated. For example, if $X$ is a ruled surface over a curve of genus greater than 1 , then its anti-canonical ring is not finitely generated in general $([\mathbf{4 9}])$. It is not a birational invariant either.

It is well known that canonical rings of algebraic curves are generated by elements of degree at most 3, and those of algebraic surfaces are expected to be generated by elements of degree at most 5 . But examples for varieties of dimension 3 show that there is no bound of degrees of generators for higher dimensional varieties even in fixed dimensions. A correct generalization for the effective statement is a birationality question. HaconMcKernan [13] and Takayama [58] proved that there exists a number $m(n)$ depending only on the dimension $n$ such that the pluricanonical system $\left|m(n) K_{X}\right|$ gives a birational map for arbitrary $n$-dimensional variety $X$ of general type. Chen-Chen [6] found an explicit and more realistic bound in the case of dimension three. See also [63] and [46] for development in this direction.

The results of the four authors [5] include the complementary case of negative Kodaira dimension where the canonical rings tell very little. The 
structure theorem as below, the existence of Mori fiber spaces, was another motivation for the MMP:

THEOREM 1.3. Let $X$ be a smooth projective variety defined over a field of characteristic 0. Assume that the canonical divisor $K_{X}$ is not pseudoeffective, i.e., not numerically equivalent to a limit of effective $\mathbf{Q}$-divisors. Then there is a birational model of $X$ which has a Mori fiber space structure.

A minimal model exists uniquely given a fixed birational class of surfaces of non-negative Kodaira dimension. But the uniqueness of minimal models fails in higher dimensions even if the Kodaira dimension is non-negative. It is proved that arbitrary birationally equivalent minimal models are connected by a sequence of operations called flops. Birationally equivalent Mori fiber spaces are connected by Sarkisov links which are generalizations of elementary transformations of ruled surfaces.

The third and more distant motivation for the MMP is a complete classification of algebraic varieties up to birational equivalence. For example, if the Kodaira dimension is zero, then the canonical ring tells very little about the structure of the variety. A minimal model of such a variety is still important and serves as a starting point of more detailed structure theory. We note that the existence of minimal models in general is still an open problem.

We always assume that the charactersitic of the base field is zero in this paper. We expect that the theorem is still true in positive characteristic, but the known proofs depend heavily on characteristic zero methods. The restriction on the characteristic should be eventually removed and the minimal model program (MMP) should work in arbitrary characteristic situation. But this restriction is necessary for the proof presented here by the two reasons:

- The resolution theorem of singularities by Hironaka is used extensively. It would be possible to extend the resolution theorem over arbitrary characteristic base or even mixed characteristic base.

- The Kodaira vanishing theorem holds true only in characteristic 0 . There are counterexamples in positive characteristic. The vanishing theorem is one of our main technical tools in every corner of the minimal model theory. In order to extend our theory to arbitrary characteristic, totally different technique should be developed.

We shall explain the following characteristic features of the MMP (cf. $[29])$ :

- inductive. The basic idea is to use the induction on the dimension. Then in more finer terms, the final product of the MMP, a minimal model or a Mori fiber space, is obtained by a step by step constructions called divisorial contractions and flips.

- logarithmic. We consider pairs consisting of varieties and boundary divisors instead of varieties alone.

- relativistic. The proof requires formulation in relative situation, i.e., we consider projective morphisms over base spaces. 
We shall explain these points in the course of the algebraic proof. It is important to note that the MMP is not yet completed because the following conjectures are still open:

- The termination conjecture of flips. This is the conjecture which is still missing in order to prove the existence of a minimal model for arbitrary algebraic variety whose canonical divisor is pseudoeffective.

- The Abundance Conjecture. We expect that some pluricanonical systems are base point free for arbitrary minimal models which are not necessarily of general type. More precisely, if $f:(X, B) \rightarrow T$ is a projective morphism from a DLT pair such that $K_{X}+B$ is nef over $T$, then $K_{X}+B$ should be semi-ample over $T$, i.e., numerically equivalent to a pull-back of an ample $\mathbf{R}$-divisor over $T$.

In particular, the following conjecture is still open: "an algebraic variety with negative Kodaira dimension is uniruled".

The organization of this paper is as follows. In order to motivate the use of the minimal models, we review a classical proof of the finite generation theorem in the case of dimension two in $\S 2$. We explain our point of view towrard minimal models, the numerical geometry, in $\S 3$ and explain how complicated problems in birational geometry are simplified to those in linear algebra. We explain how singularities of pairs appear naturally in $\S 4$, and try to justify these cumbersome definitions. The algorithm of the Minimal Model Program (MMP) is explained in $\S 5$. The main results are presented in $\S 6$ that there exists a minimal model in the case where the canonical divisor is pseudo-effective or a Mori fiber space otherwise under the additional assumption that the boundary is big. The main idea of proof is to apply the vanishing theorems for the extension problems of pluricanonical forms $(\S 7)$. The proofs of the main steps, the existence and termination of flips, are respectively surveyed in $\S 8$ and $\S 9$. The last section $\S 10$ is concerned on the non-uniqueness problem of the birational models.

\section{Case of dimension one or two: motivation of the MMP}

We start with the low dimensional cases in order to explain the idea how to use the minimal models. We note that the proofs of the finite generation theorem in dimensions one and two are valid in arbitrary characteristic.

Let us consider the case $\operatorname{dim} X=1$ and let $g$ be the genus of $X$. There are three cases:

Case 1: $g \geq 2$. Then $K_{X}$ is ample, hence the canonical ring is finitely generated.

Case 2: $g=1$. Then $K_{X}=0$, hence $R\left(X, K_{X}\right) \cong k[x]$.

Case 3: $g=0$. Then $R\left(X, K_{X}\right) \cong k$.

Therefore the canonical ring is always finitely generated in dimension one. 
The above division into cases is generalized to higher dimensions by a birational invariant called the Kodaira dimension of the pair $(X, D)$ defined by

$$
\kappa(X, D)=\text { trans.deg } R(X, D)-1
$$

if $R(X, D) \neq k$, and $\kappa(X, D)=-\infty$ otherwise. The latter convention comes from the estimate that $\operatorname{dim} H^{0}(X, m D) \sim m^{\kappa}$ for large $m . \kappa(X, D)$ can take values among $-\infty, 0,1, \ldots, \operatorname{dim} X$. In particular, we define $\kappa(X)=$ $\kappa\left(X, K_{X}\right)$. If $\kappa(X, D)=\operatorname{dim} X$, then $D$ is called big. $X$ is said to be of general type if $K_{X}$ is big.

We recall a proof by Mumford [41] (appendix to [64]) of the finite generation theorem when $\operatorname{dim} X=2$ and $X$ is of general type.

Step 1 A curve $C$ on $X$ is said to be a $(-1)$-curve if $C \cong \mathbf{P}^{1}$ and $\left(C^{2}\right)=-1$, where $\left(C^{2}\right)$ denotes the self intersection number. In other words, the normal bundle $N_{C / X}$ has degree -1 . Castelnuovo's contraction theorem tells us that there exists a birational morphism $X \rightarrow X_{1}$ to another smooth projective surface which contracts $C$ to a point. By using Castelnuovo's contraction theorem repeatedly, we obtain a birational morphism $X \rightarrow X^{\prime}$ to a minimal model on which there is no $(-1)$-curve. We note that the minimal model $X^{\prime}$ is still smooth. It is known that there exists unique minimal model $X^{\prime}$ though the order of contractions of $(-1)$-curves can be arbitrary.

Step 2 A curve $C$ on $X$ is said to be a $(-2)$-curve if $C \cong \mathbf{P}^{1}$ and $\left(C^{2}\right)=-2$. Artin's contraction theorem $([\mathbf{3}])$ states that all the $(-2)$ curves can be contracted to points by a birational morphism $X^{\prime} \rightarrow X^{\prime \prime}$ to the canonical model. The canonical model $X^{\prime \prime}$ is a projective surface with isolated rational singularities called Du Val singularities or canonical singularities. Though $X^{\prime \prime}$ is singular, we have still define a canonical divisor $K_{X^{\prime \prime}}$ as a Cartier divisor, hence the canonical ring $R\left(X^{\prime \prime}, K_{X^{\prime \prime}}\right)$.

Step 3 The canonical ring is invariant under the contractions:

$$
R\left(X, K_{X}\right) \cong R\left(X^{\prime}, K_{X^{\prime}}\right) \cong R\left(X^{\prime \prime}, K_{X^{\prime \prime}}\right) .
$$

$K_{X^{\prime \prime}}$ is ample, hence $R\left(X, K_{X}\right)$ is finitely generated.

Step 1 will be generalized to the MMP, and Step 2 to the Base Point Free Theorem.

A proof when $\operatorname{dim} X=2$ and $\kappa=1$ uses Kodaira's theory of elliptic surfaces $([\mathbf{3 2}])$.

Step 1 is the same as above. Let $X^{\prime}$ be a minimal model of $X$. It is unique again.

Step 2 By the Enriques classification, there is an elliptic surface structure $f: X^{\prime} \rightarrow Y ; Y$ is a smooth projective curve and general fibers of $f$ are elliptic curves. There may be degenerate fibers called singular fibers.

Step 3 We define a Q-divisor, a divisor with rational coefficients, $B_{Y}=\sum_{i} b_{i} B_{i}$ on $Y$ which measures the degeneration of the morphism 
$f$. The coefficients $b_{i}$ belong to $\frac{1}{12} \mathbf{Z}$, corresponding to the fact that there exists an automorphic form of degree 12 . The values of the $b_{i}$ are determined by Kodaira's classification of singular fibers ([32]). If a singular fiber of $f$ has finite local monodromy, then the corresponding coefficient belong to the interval $(0,1)$. Otherwise, the natural coefficient can be arbitrarily large, but we can move the divisor $B_{Y}$ to another Q-divisor $B_{Y}^{\prime}$ by using the automorphic form so that all the coefficients are less than 1 . Thus we obtain the following canonical bundle formula of Kodaira:

$$
K_{X^{\prime}}=f^{*}\left(K_{Y}+B_{Y}^{\prime}\right) \text {. }
$$

Therefore the canonical ring of $X$ is isomorphic to the $\log$ canonical ring for a lower dimensional pair $\left(Y, B_{Y}^{\prime}\right)$ :

$$
R\left(X, K_{X}\right) \cong R\left(X^{\prime}, K_{X^{\prime}}\right) \cong R\left(Y, K_{Y}+B_{Y}^{\prime}\right)
$$

We note that the concept of the section ring is naturally extended to the case where the coefficients are no more integers as follows:

$$
R(X, D)=\bigoplus_{m=0}^{\infty} H^{0}(X,\llcorner m D\lrcorner)
$$

where $\llcorner m D\lrcorner$ denotes the round down of $m D$ obtained by taking the round downs of the coefficients. Since $K_{Y}+B_{Y}^{\prime}$ is ample, we have the finite generatedness of the canonical ring again.

This is one of the motivations of the "log" theory which will be explained later.

The case $\operatorname{dim} X=2$ and $\kappa=0$ is easy; we have $R\left(X, K_{X}\right) \cong k[x]$.

The classification of surfaces tells us that a minimal model of such a variety, which is unique again, is isomorphic to either an abelian surface, a K3 surface, or their quotient. We note that the higher dimensional generalizations of this class include interesting varieties such as Calabi-Yau manifolds and hyperKähler manifolds.

In order to explain the second motivation for the MMP, we consider the case where $\kappa(X)=-\infty$. In this case, the canonical ring is trivial and tells very little. Instead there is an explicit structure theorem when $\operatorname{dim} X=2$ and $\kappa(X)=-\infty$.

Step 1 is the same. Let $X^{\prime}$ be a minimal model of $X$.

Step 2 There are two cases according to the Enriques classification. The first case is a ruled surface $f: X^{\prime} \rightarrow Y ; Y$ is a smooth projective curve and all fibers are isomorphic to $\mathbf{P}^{1}$. In the second case, we have $X^{\prime} \cong \mathbf{P}^{2}$.

Step 2 will be generalized to a Mori fiber space in the MMP explained later. This is another motivation for the MMP.

A minimal model $X^{\prime}$ of an algebraic surface $X$ with $\kappa(X) \geq 0$ is uniquely determined by the birational class of $X$ and is independent of the contraction process of $(-1)$-curves. On the other hand, there are many minimal models in the sense that there are no $(-1)$-curves if $\kappa(X)=-\infty$. It is known 
that birationally equivalent minimal models are connected by a sequence of elementary transformations.

There is another approach toward the finite generation theorem using the Zariski decomposition $([\mathbf{6 4}])$. In the case of a surface $X$, there is a birational morphism $f: X \rightarrow X^{\prime}$ to its minimal model. We can write $K_{X}=f^{*} K_{X}+E$, where $E$ is an effective divisor, a divisor with non-negative coefficients, whose support coincides with the exceptional locus, the locus where $f$ is not an isomorphism. The point is that this decomposition can be constructed on $X$ without using the minimal model provided that $\kappa(X) \geq 0$.

In general, if $D$ is an effective divisor on a smooth projective surface $X$, then there exist uniquely determined Q-divisors $P$ and $E$, divisors with coefficients in $\mathbf{Q}$, which satisfy the following conditions:

(1) $P$ is nef, i.e., the intersection numbers $(P \cdot C)$ are non-negative for all curves $C$ on $X$.

(2) $E$ is effective.

(3) If $\left\{E_{1}, \ldots, E_{t}\right\}$ is the set of irreducible components of $E$, then $\left(P \cdot E_{i}\right)=0$ for all $i$ and the matrix $\left[\left(E_{i} \cdot E_{j}\right)\right]$ is negative definite.

For example, $K_{X}=f^{*} K_{X}+E$ is the Zariski decomposition of the canonical divisor which is regarded as an effective $\mathbf{Q}$-divisor when $m K_{X}$ is effective for a positive integer $m$. Therefore a minimal model is virtually obtained as the nef part of the canonical divisor.

We obtained a minimal model of a log surface by using the Zariski decomposition in the paper [18] as a log generalization of the minimal model theory in dimension two. Let $X$ be a smooth projective variety of dimension two and $B$ a reduced normal crossing divisor. Then the Zariski decomposition $K_{X}+B=P+E$ gives a minimal model of the pair $(X, B)$; the support of $E$ can be contracted to points by a birational morphism $f: X \rightarrow X^{\prime}$ to a normal surface and we have $P=f^{*} K_{X^{\prime}}$. We note that the minimal model $X^{\prime}$ may have singularities, called log terminal singularities, and that the coefficients of $P$ are not necessarily integers even if those of $B$ are equal to one. Moreover we can prove that a linear system $|m P|$ is base point free for a positive integer $m$.

There is a higher dimensional generalization of the concept of the Zariski decomposition. The technique of the base point free theorem explained later implies that the finite generation theorem holds if there exists a Zariski decomposition of a $(\log )$ canonical divisor $([\mathbf{2 1}, \mathbf{4 0}])$. But there is a counterexample for the existence of the Zariski decomposition if we consider arbitrary effective divisors $([44])$. On the other hand, the Zariski decomposition of a log canonical divisor should exist because the log canonical divisor of a minimal model will give the Zariski decomposition. The nonuniqueness of minimal models and the uniqueness of the Zariski decomposition are compatible, because birationally equivalent minimal models have equivalent canonical divisors. 
We note that the Zariski decomposition can be achieved only on a blown-up variety of the original model if it ever exists; this is a phenomenon in dimension three or higher due to the existence of flips. The point is to resolve simultaneously the base loci of all the pluricanonical systems on a fixed variety. Such simultaneous resolution can be considered on the Zariski space, the inverse limit of blowing up sequences. The b-divisors by Shokurov [53] is a formal concept to consider such situations. The polytope decomposition theorem explained later will solve this infinity problem and lead to the flip theorem.

There is also a complex analytic version of the Zariski decomposition by Tsuji $([\mathbf{6 0}, \mathbf{9}])$. It's existence is rather easily proved though it is not as strong as the algebraic counterpart.

\section{Numerical geometry}

We would like to generalize the minimal model theory to higher dimensional varieties. The first task is to generalize contraction theorems of Castelnuovo and Artin. It is a highly non-linear problem to identify the locus to be contracted and to construct a contraction morphism. A linearization of this problem is achieved by using the intersection numbers of divisors with curves. The method is called numerical geometry. Please distinguish it from enumerative geometry. The idea to use cones in real vector spaces in order for the investigation of the birational geometry goes back to Hironaka's thesis [16].

We consider a relative situation; let $X$ be an algebraic variety which is projective over a base space $T$. If the base space $T$ is a point (absolute case), then $X$ is just a projective variety. We shall need to consider more general base space $T$ in order to use inductive arguments. Although the descriptions of the relative situations are slightly longer, the same methods of proofs work as in the absolute case.

We assume that $X$ is normal. Let $D=\sum_{i} d_{i} D_{i}$ be an $\mathbf{R}$-divisor, a formal linear combination of prime divisors $D_{i}$, reduced irreducible subvarieties of codimension one, with coefficients $d_{i}$ in $\mathbf{R}$. It is said to be an $\mathbf{R}$-Cartier divisor if it can be expressed as a linear combination of Cartier divisors with coefficients in $\mathbf{R}$. As a counterpart, let $C=\sum_{i} c_{i} C_{i}$ be an $\mathbf{R}$-1-cycle, a formal linear combination of curves $C_{i}$, reduced irreducible subvarieties of dimension one, with coefficients $c_{i}$ in $\mathbf{R}$. It is said to be relative for $f$ if the $C_{i}$ are mapped to points of the base space. Then we can define a bilinear pairing called the intersection number $(D \cdot C)$ with values in $\mathbf{R}$ if $D$ is $\mathbf{R}$-Cartier and $C$ is relative for $f$. Two $\mathbf{R}$-Cartier divisors or two relative $\mathbf{R}$-1-cycles are said to be numerically equivalent if they give the same intersection numbers when paired to arbitrary counterparts. We note that we cannot define the intersection number of an arbitrary $\mathbf{R}$-divisor which is not $\mathbf{R}$-Cartier with a curve. 
We consider real vector spaces

$$
\begin{aligned}
& N^{1}(X / T)=\{\mathbf{R} \text {-Cartier divisor }\} / \text { numerical equivalence } \\
& N_{1}(X / T)=\{\text { relative } \mathbf{R} \text {-1-cycle }\} / \text { numerical equivalence }
\end{aligned}
$$

which are dual to each other and known to be finite dimensional. The dimension $\rho(X / T)=\operatorname{dim} N^{1}(X / T)$ is called the Picard number of $X$ over $T$. It is an important numerical invariant next to the $\operatorname{dimension} \operatorname{dim} X$.

Let $\overline{N E}(X / T)$ be the closed convex cone in $N_{1}(X / T)$ generated by the numerical classes of relative curves, and let $\operatorname{Nef}(X / T)$ be the nef cone defined as the dual closed convex cone in $N^{1}(X / T)$ :

$$
\operatorname{Nef}(X / T)=\left\{v \in N^{1}(X / T) \mid(v \cdot C) \geq 0 \forall C\right\}
$$

where the $C$ are arbitrary curves relative for $f$. An $\mathbf{R}$-divisor is called relatively nef, nef over $T$, or $f$-nef, if its numerical class belongs to $\operatorname{Nef}(X / T)$. The following Kleiman's criterion is fundamental ([30]):

TheOREm 3.1. A Cartier divisor $D$ is relatively ample over $T$ if and only if its numerical class belongs to the interior $A m p(X / T)$ of the nef cone $\operatorname{Nef}(X / T)$ called the ample cone.

An $\mathbf{R}$-Cartier divisor $D$ is said to be ample over $T$, or $f$-ample, if its numerical class belongs to the ample cone $\operatorname{Amp}(X / T)$, i.e., if it is a linear combination of ample Cartier divisors with positive linear coefficients. We note that an ample R-Cartier divisor does not in general become a Cartier divisor by multiplying a positive number and it is not directly related to a projective embedding.

An $\mathbf{R}$-Cartier divisor $D$ is said to be big over $T$, or $f$-big, if one can write $D=A+E$ for an $f$-ample $\mathbf{R}$-Cartier divisor $A$ and an effective $\mathbf{R}$-divisor $E$, an $\mathbf{R}$-divisor with non-negative coefficients. In other words, an $\mathbf{R}$-divisor is big if it is bigger than an ample divisor. The set $\operatorname{Big}(X / T)$ of numerical classes of all the $f$-big $\mathbf{R}$-divisors is an open cone in $N^{1}(X / T)$ called the big cone. $D$ is said to be pseudo-effective over $T$ if its numerical class belongs to the the closure $\operatorname{Psef}(X / T)$ of the big cone callled a pseudo-effective cone. In other words, $D$ is pseudo-effective if its numerical class is a limit of effective Q-divisors. We have the following commutative diagram of inclusions:

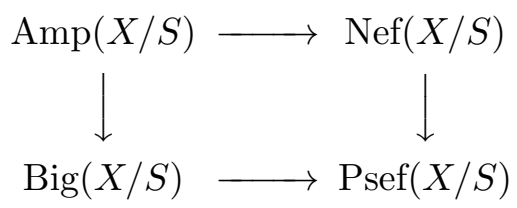

where the cones on the left are open and on the right their closures.

Let $f: X \rightarrow Y$ be an arbitrary surjective morphism to another normal variety which is projective over $T$ such that all the geometric fibers of $f$ are connected. Then the natural homomorphism $f^{*}: N^{1}(Y / T) \rightarrow N^{1}(X / T)$ is injective, and the image of the nef cone satisfies

$$
f^{*} \operatorname{Nef}(Y / T)=\operatorname{Nef}(X / T) \cap f^{*} N^{1}(Y / T) .
$$


If $f$ is not an isomorphism, then $F=f^{*} \operatorname{Nef}(Y / T)$ is a face of $\operatorname{Nef}(X / T)$ in the sense that $F \subset \partial \operatorname{Nef}(X / T)$, because the pull-back of an ample divisor on $Y$ is never ample on $X$. There is a dual face $F^{*}$ of $\overline{N E}(X / T)$ defined by

$$
F^{*}=\left\{w \in \overline{N E}(X / T) \mid f_{*} w=0\right\} .
$$

Indeed we have

$$
F^{*}=\{w \in \overline{N E}(X / T) \mid(D \cdot w)=0 \forall D\}
$$

where the $D$ are arbitrary ample divisors on $Y$. By the Zariski main theorem, the morphism $f$ is uniquely determined by the face $F$ or $F^{*}$ as long as $Y$ is normal.

The converse is not true; there are faces which do not correspond to any morphisms. The point of the MMP is that there always exists a morphism if the face $F^{*}$ on the cone of curves lies in the negative side for the log canonical divisor, i.e., if the intersection numbers with the log canonical divisor are negative for all non-zero vectors in $F^{*}$.

In the case where $X$ is smooth and $T$ is a point, Mori proved in his famous paper [38] that, if the canonical divisor $K_{X}$ is not nef, then there always exists an extremal ray, a face $F^{*}$ with $\operatorname{dim} F^{*}=1$ on which $K_{X}$ is negative. Moreover he proved that there exists a corresponding contraction morphism from $X$ in the case $\operatorname{dim} X=3$. These results were generalized to the cone and contraction theorems explained later.

The key point in $[\mathbf{3 8}]$ is to prove that there exists a rational curve which generates an extremal ray $F^{*}$. In order to prove this, he employed the deformation theory over a base field of positive characteristic, a purely algebraic method. It is remarkable that this is still the only method to prove the existence of rational curves; there is no purely characteristic zero proof nor complex analytic proof. The result is generalized to arbitrary pairs with log terminal singularities in [22] using [37]; an extremal ray is always generated by a rational curve.

In this paper we shall only deal with cones in $N^{1}(X / T)$ and not consider those in $N_{1}(X / T)$. The reason will be clear in the polytope decomposition theorem.

\section{Log canonical divisors and log terminal singularities}

In order to extend the concept of the minimality to higher dimensional varieties, we have to change the point of view. We look at canonical divisors instead of varieties themselves. Moreover, we have to deal with logarithmic pairs instead of varieties. Singularities appear naturally in this way.

We explain how to compare canonical divisors of birationally equivalent varieties as a way to compare different birational models. Let $\alpha: X \rightarrow X^{\prime}$ be a birational map between smooth projective varieties. Then there exists a third smooth projective variety $X^{\prime \prime}$ with birational morphisms $f: X^{\prime \prime} \rightarrow X$ and $f^{\prime}: X^{\prime \prime} \rightarrow X^{\prime}$ such that $\alpha=f^{\prime} \circ f^{-1}$. If we define canonical divisors $K_{X}$ and $K_{X^{\prime}}$ by using the same rational differential $n$-form, then the difference 
$f^{*} K_{X}-\left(f^{\prime}\right)^{*} K_{X^{\prime}}$ is a well defined divisor which is supported above the locus where $\alpha$ is not an isomorphism. Moreover, it is independent of the choice of the rational differential $n$-form which was used to define $K_{X}$ and $K_{X^{\prime}}$. We define that an equality $K_{X} \geq K_{X^{\prime}}$ holds if and only if their pull-backs satisfy $f^{*} K_{X} \geq\left(f^{\prime}\right)^{*} K_{X^{\prime}}$.

Let us consider some examples before stating a formal definition of log terminal singularities. If $f: X \rightarrow X^{\prime}$ is a contraction morphism of a (-1)curve $C$, then $K_{X}-C=f^{*} K_{X^{\prime}}$. An important observation is that the canoical divisor becomes smaller when the variety becomes smaller. Therefore, we come to the following (temporary) defeinition: A variety $X$ is said to be a minimal model if $K_{X}$ is minimal among all birationally equivalent varieties.

We note that, if $f: X \rightarrow X^{\prime}$ is a contraction morphism of (-2)-curves, then $K_{X}=f^{*} K_{X^{\prime}}$ and $X$ has canonical singularities.

Let us consider an example in higher dimension. Let $X$ be a smooth variety of dimension $n$ and $E$ a prime divisor which is isomorphic to $\mathbf{P}^{n-1}$ and such that the normal bundle $N_{E / X}$ is isomorphic to $\mathcal{O}_{\mathbf{P}^{n-1}}(-d)$ for a positive integer $d$. Then there exists a birational morphism $f: X \rightarrow X^{\prime}$ which contracts $E$ to a point. If $d \geq 2$, then $X^{\prime}$ has an isolated quotient singularity, a typical example of a log terminal singularity. We can still define a canonical divisor $K_{X^{\prime}}$ as a $\mathbf{Q}$-Cartier divisor, namely $d K_{X^{\prime}}$ is a Cartier divisor. We have an equation

$$
K_{X}+\left(1-\frac{n}{d}\right) E=f^{*} K_{X^{\prime}}
$$

Thus $K_{X}>K_{X^{\prime}}$ if $n>d$. In this case, the singularity of $X^{\prime}$ is said to be terminal. For example, if $n=3$ and $d=2$, then $K_{X}>K_{X^{\prime}}$. This terminal singularity was first discovered by Mori [38].

$d$ is the smallest positive integer for which $d K_{X^{\prime}}$ becomes a Cartier divisor. Such an integer is called a Cartier index of the singularity. Therefore there exists an isolated quotient singularity in dimension three which is terminal and has arbitrarily large Cartier index. This is the reason why there is no bound of degrees for the generators of canonical rings in dimension three. Indeed let $X$ be a projective variety having only terminal singularities such that there is a point at which the Cartier index is $d$ and that the canonical divisor $K_{X}$ is ample. Then the canonical ring of $X$ is not generated by elements whose degrees are less than $d$.

We have to consider the log version of a canonical divisor, i.e., the $l o g$ canonical divisor $K_{X}+B$ of a log pair $(X, B)$ in the MMP. There are many reasons which lead us to consider the log version:

- The canonical divisor of a fiber space is described by using a log canonical divisor as explained already in the case of elliptic surfaces. The canonical divisor of a finite covering is similarly described.

- The adjunction formula for log canonical divisors is the basic tool for the inductive argument on dimensions. 
- The natural range for the power of vanishing theorems is the category of log varieties.

An objact of the log theory is a pair $(X, B)$ consisting of a normal variety $X$ and an $\mathbf{R}$-divisor $B$ called a boundary. In many cases, we assume that $B$ is effective, i.e., the coefficients are non-negative. If $B$ is not effective, we often call $B$ a subboundary in order to distinguish from the effective case. The coefficients of $B$ are not necessarily integers, but rational or even real numbers. The rational coefficients naturally appear when we consider positive multiples of divisors, while the real coefficients when taking the limits. The canonical divisor $K_{X}$ is still defined as a Weil divisor as long as the variety $X$ is normal. The $\log$ canonical divisor is the sum $K_{X}+$ $B$. For example, if $X$ is smooth and $B$ is a reduced divisor, i.e., all the coefficients are equal to 1, with normal crossing support, then $K_{X}+B$ is the divisor corresponding to the determinant line bundle of logarithmic differential forms $\Omega_{X}^{1}(\log B)$. This is the origin of the name "log".

We note that our log structures are different from the log structures of Fontaine-Illusie-Kato [17]. Both concepts are derived as generalizations of normal crossing divisors on smooth varieties. There is no log theory which includes both at the moment.

We have to introduce singularities in higher dimensions by two resasons. The first is that the canoical divisor can become smaller when the variety acquires singularities in the case $\operatorname{dim} X \geq 3$ as observed in the previous example. The other is that we have to consider pairs of varieties and divisors, i.e., $\log$ varieties.

Now we state a formal definition:

Definition 4.1. Let $X$ be a normal variety. It is said to be $\mathbf{Q}$-factorial if an arbitrary prime divisor on $X$ is a $\mathbf{Q}$-Cartier divisor, a $\mathbf{Q}$-linear combination of Cartier divisors.

Let $(X, B)$ be a pair of a normal variety $X$ and an $\mathbf{R}$-divisor $B=$ $\sum_{i} b_{i} B_{i}$, and let $\mu: Y \rightarrow X$ be a projective birational morphism from another variety. The exceptional locus of $\mu$ is the smallest closed subset of $Y$ such that $\mu$ is an isomorphism when restricted on its complement. $\mu$ is said to be a $\log$ resolution (in a strict sense) of the pair $(X, B)$, if $Y$ is smooth, the exceptional locus is a normal crossing divisor, and moreover the union of the exceptional locus and the strict transforms $\mu_{*}^{-1} B_{i}$ of the prime divisors $B_{i}$ is a normal crossing divisor.

The pair $(X, B)$ is said to be divisorially log terminal (DLT) or to have only divisorially log terminal singularities if there exists a log resolution such that the following conditions are satisfied:

(1) The coefficients $b_{i}$ belong to the interval $(0,1]$.

(2) The log canonical divisor $K_{X}+B$ is an $\mathbf{R}$-Cartier divisor.

(3) We can write

$$
\mu^{*}\left(K_{X}+B\right)=K_{Y}+\mu_{*}^{-1} B+\sum d_{j} D_{j}
$$


with $d_{j}<1$ for all $j$, where $\mu_{*}^{-1} B=\sum b_{i} \mu_{*}^{-1} B_{i}$ is the strict transform of $B$ and the $D_{j}$ are prime divisors which are contained in the exceptional locus of $\mu$.

If $0<b_{i}<1$ for all $i$ instead of the condition 1 , then the pair is called $K L T$. In other words, we can write

$$
\mu^{*}\left(K_{X}+B\right)=K_{Y}+B_{Y}
$$

where the coefficients of $B_{Y}$ belong the interval $(0,1)$. The pair is said to be $\log$ canonial $(L C)$ if all the coefficients of $B_{Y}$ belong to the interval $(0,1]$ in the above formula.

If $d_{j} \leq 0$ or $d_{j}<0$ for all $j$ instead of the condition 3 , then the pair is called canonical or terminal, respectively. We usually assume that $B=0$ for canonical or terminal singularities, but those with $B \neq 0$ are also useful.

For example, if $X$ is smooth and the support of $B$ is a normal crossing divisor, then the pair $(X, B)$ is DLT (resp. KLT) if and only if the coefficients are in $(0,1]$ (resp. $(0,1))$.

The condition 2 is necessary for definig the pull-back in the condition 3. The point of the log terminality is that $K_{X}+B$ is strictly smaller than $K_{Y}+\mu_{*}^{-1} B+\sum D_{j}$. In other words, the pair is locally minimal among all birational models in the log sense. DLT and KLT pairs are respectively called weal log terminal and log terminal in [29].

The log canonical singularites do not behave so well as log terminal singularities. For example, the underlying variety $X$ may have non CohenMacaulay singularities. We can compare this with the fact that the underlying variety of a DLT pair has always rational singularities in characteristic zero. But we have often a situation where there exists another boundary $B^{\prime}$ on an LC pair $(X, B)$ such that $\left(X, B^{\prime}\right)$ is KLT. In this case there is no problem because we can easily extend arguments in the MMP by usnig the perturbation to a new boundary $(1-\epsilon) B+\epsilon B^{\prime}$.

To be KLT is an open condition under varying coefficients of the boundary, LC is closed, and DLT is intermediate. Each of them has advantage and disadvantage. We can use a compactness argument for the LC pairs.

The KLT condition is equivalent to the $L^{2}$ condition in complex analysis. This stronger version of the log terminality is easier to handle and more natural in some cases. For example, KLT condition is independent of a log resolution. Moreover, some statements are only true for KLT pairs. On the other hand, it is necessary to consider general DLT pairs for the induction argument on the dimension. Indeed the adjunction formula holds only along a boundary component with coefficient one.

The pairs having $\mathbf{Q}$-factorial DLT singularities can be characterized as those which may appear in the process of the MMP if we start with pairs of smooth varieties and reduced normal crossing divisors. This is the category of pairs we work in the MMP. 


\section{Minimal Model Program (MMP)}

We explain the algorithm of the MMP in this section. There are some standard references including $[29,33,35,36]$ and $[8]$.

The cone and the contraction theorems are fundamental for the MMP. As we already explained, some of the faces of the nef cone correspond to morphisms while some are not. Mori's discovery in $[\mathbf{3 8}]$ is that, if the dual face of the cone of curves is contained in the half space on which the canonical divisor is negative, then there should always exist a corresponding morphism. In particular we define an extremal ray to be such a one dimensional face of the cone of curves.

Now we state the cone and contraction theorems for the log pairs $([\mathbf{2 9}])$. We state the theorem in the dual terminology on the cone of divisors:

TheOREM 5.1 (Cone Theorem). Let $f:(X, B) \rightarrow T$ be a projective morphism from a DLT pair, $H$ an ample divisor for $f$, and $\epsilon$ a positive number. Then the part of the nef cone $\operatorname{Nef}(X / T)$ which is visible from the point $\left[K_{X}+B+\epsilon H\right] \in N^{1}(X . T)$ is generated by finitely many points whose coordinates are rational numbers.

If $K_{X}+B$ is nef, then the assertion is empty. If $K_{X}+B$ is not nef, then $K_{X}+B+\epsilon H$ is not nef either if $\epsilon$ is sufficiently small. The Come Theorem says that the boundary of the nef cone facing the point $\left[K_{X}+B+\epsilon H\right]$ is like a boundary of a polyhedral cone, and consists of finitely many rational faces, intersections of $\operatorname{Nef}(X / T)$ with linear subspaces of $N^{1}(X / T)$. When the positive number $\epsilon$ approaches to zero, the number of visible faces increases, so that there may be eventually infinitely many faces.

TheOREM 5.2 (Contraction Theorem). Let $f:(X, B) \rightarrow T$ be a projective morphism from a DLT pair, and let $F \subset N e f(X / T)$ be a face which is visible from the point $\left[K_{X}+B+\epsilon H\right]$ as above. Then there exists a surjective morphism $\phi: X \rightarrow Y$ to a normal variety which is projective over the base space $T$ such that the geometric fibers of $\phi$ are connected and that

$$
F=\phi^{*} N e f(Y / T)
$$

In particular, if the codimension of the face $F$ is equal to one, then we call $\phi$ a primitive contraction.

The Contraction Theorem is equivalent to the Base Point Free Theorem:

Theorem 5.3 (Base Point Free Theorem). Let $f:(X, B) \rightarrow T$ be a projective morphism from a DLT pair, and let $D$ be a Cartier divisor on $X$. Assume that $D$ is $f$-nef and that $D-\epsilon\left(K_{X}+B\right)$ is $f$-ample for a small positive number $\epsilon$. Then there exists a positive integer $m_{0}$ such that $m D$ is relatively base point free for arbitrary $m \geq m_{0}$.

The conclusion is saying that the natural homomorphism

$$
f^{*} f_{*} \mathcal{O}_{X}(m D) \rightarrow \mathcal{O}_{X}(m D)
$$


is surjective. The Base Point Free Theorem implies in particular the Non-Vanishing Theorem which states that $f_{*} \mathcal{O}_{X}(m D) \neq 0$.

We explain steps of the MMP formulated by Reid $([48])$ modified to the $\log$ and relative situation. The coefficients of the boundaries are real numbers, so that the limiting arguments are made possible.

We consider the category of $\mathbf{Q}$-factorial $D L T$ pairs $(X, B)$ consisting of varieties $X$ which are projective over a base space $T$ together with $\mathbf{R}$-divisors $B$. Let $f: X \rightarrow T$ be the structure morphism. This category includes pairs consisting of smooth varieties and normal crossing divisors with coefficients belonging to in the interval $(0,1]$.

Case 1: If $K_{X}+B$ is relatively nef over the base (i.e., $f$-nef), then $(X, B)$ is minimal. We stop here in this case.

Case 2: If $K_{X}+B$ is not relatively nef, then there exists a primitive contraction morphism $\phi: X \rightarrow Y$ by the Cone and Contraction Theorems. It is important to note that we need to choose one of the codimension one faces of the nef cone.

There are three cases for $\phi$.

- If $\operatorname{dim} X>\operatorname{dim} Y$, then $\phi$ is called a Mori fiber space. We stop here in this case.

- If $\phi$ is a birational morphism and contracts a divisor, i.e, if $\phi$ is a divisorial contraction, then the exceptional locus of $\phi$ is proved to be a prime divisor thanks to the $\mathbf{Q}$-factoriality condition. The new pair $\left(X^{\prime}, B^{\prime}\right)=\left(Y, \phi_{*} B\right)$ is again $\mathbf{Q}$-factorial and DLT. We go back to Case 1 or 2 , and continue the program.

- If $\phi$ is small, i.e., if $\phi$ changes the variety only in codimension two or higher, then there exists another small projective birational morphism $\phi^{\prime}: X^{\prime} \rightarrow Y$ for which $K_{X^{\prime}}+B^{\prime}$ is positive where $B^{\prime}$ is the strict transform of $B$. We note that the set of prime divisors stay unchanged under the birational map $\left(\phi^{\prime}\right)^{-1} \circ \phi$ which is called a flip. The existence of a flip is now a theorem, called the Flip Theorem, by Hacon and McKernan [14]. The new pair $\left(X^{\prime}, B^{\prime}\right)$ is again $\mathbf{Q}$-factorial and DLT. We can continue the program.

We should prove that there does not exist an infinite chain of flips in order to obtain the final product, a minimal model or a Mori fiber space. This Termination of Flips is still a conjecture in the general case.

It is important to note that an inequality $K_{X}+B>K_{X^{\prime}}+B^{\prime}$ always holds after a divisorial contraction or a flip.

The Picard number drops by one after a divisorial contraction: $\rho(Y / T)=$ $\rho(X / T)-1$. Therefore the number of possible divisorial contractions is bounded by the Picard number.

But the Picard number stays the same after a flip. Indeed there is a natural isomorphism $\left(\phi_{*}^{\prime}\right)^{-1} \circ \phi_{*}: N^{1}(X / T) \rightarrow N^{1}\left(X^{\prime} / T\right)$. Under the 
identification of real vector spaces by this isomorphism, the nef cones $\operatorname{Nef}(X / T)$ and $\operatorname{Nef}\left(X^{\prime} / T\right)$ are adjacent and touch each other along the face $F$, the pull-back of the nef cone $\operatorname{Nef}(Y / T)$. Therefore we can investigate the changes of birational models by looking at a partial cone decomposition of the real vector space.

ExAMPLE 5.4. The following is an example of a flip called Francia's flip [10]. This example was first considered as a counterexample to the existence of a minimal model in higher dimension. Now it is incorporated into the MMP.

We have $\operatorname{dim} X=3$ and $B=0$ in this example. Let $X^{\prime}$ be a smooth projective variety containing a smooth rational curve $C^{\prime}$, i.e., a curve isomorphic to $\mathbf{P}^{1}$, whose normal bundle is isomorphic to $\mathcal{O}_{\mathbf{P}^{1}}(-1) \oplus \mathcal{O}_{\mathbf{P}^{1}}(-2)$. By blowing-up and blowing-down, we can construct a normal projective variety $X$ with an isolated quotient singularity $P$ and a smooth rational curve $C$ on $X$ passing through $P$ such that $X^{\prime} \backslash C^{\prime}$ is isomorphic to $X \backslash C$.

There is a small contraction morphism $\phi: X \rightarrow Y$ which contracts $C$ to a point $Q . K_{X}$ is negative for $\phi ;\left(K_{X} \cdot C\right)=-\frac{1}{2}<0$. There is a small biratiotnal morphism $\phi^{\prime}: X^{\prime} \rightarrow Y$ on the other side such that $\phi^{\prime}\left(C^{\prime}\right)=Q$. $K_{X^{\prime}}$ is positive for $\phi^{\prime} ;\left(K_{X^{\prime}} \cdot C^{\prime}\right)=1$.

The MMP is a way to obtain a minimal model. We note that this is not the only way. We give a formal definition of a minimal model and a canonical model:

Definition 5.5. Let $\left(X_{0}, B_{0}\right)$ be an LC pair which is projective over a base $T$. We assume that there exists another boundary $B_{0}^{\prime}$ such that $\left(X_{0}, B_{0}^{\prime}\right)$ is KLT. A new $\mathrm{Q}$-factorial LC pair $(X, B)$ projective over a base $T$ together with a birational map $\alpha: X_{0} \rightarrow X$ over $T$ is said to be a minimal model of $\left(X_{0}, B_{0}\right)$ if the following conditions are satisfied:

(1) $\alpha_{0}$ is surjective in codimension one, i.e., an arbitrary codimension one scheme theoretic point of $X$ is in the image of a morphism which represents $\alpha$.

(2) $B=\alpha_{*} B_{0}$ is the strict transform.

(3) If $\mu_{0}: Y \rightarrow X_{0}$ and $\mu: Y \rightarrow X$ are common resolutions, then the difference $\mu_{0}^{*}\left(K_{X_{0}}+B_{0}\right)-\mu^{*}\left(K_{X}+B\right)$ is effective. Moreover arbitrary codimension one point of $Y$ which remains codimension one on $X_{0}$ but not on $X$ is contained in the support of the difference $\mu_{0}^{*}\left(K_{X_{0}}+B_{0}\right)-\mu^{*}\left(K_{X}+B\right)$.

A surjective morphism $f: X \rightarrow Z$ with connected geometric fibers from a minimal model to a normal variety projective over $T$ is said to be the canonical model if $K_{X}+B$ is numerically equivalent to the pull-back $f^{*} H$ for a relatively ample $\mathbf{R}$-divisor $H$ on $Z$.

The triple $(X, B, \alpha)$ is more precisely called a marked minimal model. The last condition for the minimal model means that a prime divisor is 
contracted by $\alpha$ only if there is a reason to be contracted. The elliptic fibration considered in $\S 2$ is an example of a canonical model.

The Base Point Free Theorem implies that if the boundary $B$ of a minimal model $(X, B)$ is big over $T$, then a canonical model always exists. The existence of a canonical model in general is a conjecture called the Abundance Conjecture.

A minimal model of a given pair may not be unique if it ever exists. But they are always equivalent; if $(X, B)$ and $\left(X^{\prime}, B^{\prime}\right)$ are two minimal models of a pair $\left(X_{0}, B_{0}\right)$ over $T$, and if $\mu: Y \rightarrow X$ and $\mu^{\prime}: Y \rightarrow X^{\prime}$ are common resolutions, then we have $\mu^{*}\left(K_{X}+B\right)=\left(\mu^{\prime}\right)^{*}\left(K_{X^{\prime}}+B^{\prime}\right)$. It follows that a canonical model is unique if it exists.

We shall need a modified version of the MMP, called the MMP with scaling or the directed $M M P$. It is much easier version than the general MMP.

The process of the MMP is not unique because we should choose a face in each step. The scaled version of the MMP has smaller ambiguity of the choice. The termination conjecture for arbitrary sequence of flips is in fact not necessary in order to prove the existence of a minimal model or a Mori fiber space. What we have to prove is that some sequence of flips terminates. It turned out that the termination conjecture is easier for the sequence of flips which is directed by an additional divisor. We can expect that a sequence of flips has more tendency to terminate when the boundary moves along a line segment in the space of divisors.

We take an additional effective $\mathbf{R}$-Cartier divisor $H$ besides the pair $(X, B)$ such that $(X, B+H)$ is still DLT. We assume that $K_{X}+B+H$ is $f$-nef. Let

$$
t_{1}=\min \left\{t \in \mathbf{R}_{\geq 0} \mid K_{X}+B+t H \text { is } f \text {-nef }\right\} \in[0,1] .
$$

If $K_{X}+B$ is not $f$-nef, then we choose a face $F$ such that $\left[K_{X}+B+t_{1} H\right] \in F$. The existence of such a face is clear in the case where $B$ is big. In the general case, the existence is proved by Birkar [4] using the boundedness of extremal rays $[\mathbf{2 2}]$.

We perform a divisorial contraction or a flip to the pair $(X, B)$. Let us denote by $(X, B)$ and $H$ the new pair and the strict transform of $H$ by abuse of language. Then the threshold $t_{1}$ for the new pair decreases or stays the same. In other words, the MMP is directed by the scaling $H$. If it reaches to 0 , then $K_{X}+B$ becomes $f$-nef, so we are done.

The point is that the log pair $\left(X, B+t_{1} H\right)$ is already minimal, since $K_{X}+B+t_{1} H$ is $f$-nef, though it is an intermediate model of the MMP for $(X, B)$. It will be proved, in the course of inductive argument, that the set of underlying varieties of minimal models is finite when we move the coefficients of the boundary on the line segment joining $B$ and $B+H$ as above. This is the Polytope Decomposition Theorem. We shall apply this theorem to the MMP with scaling for the termination argument. 


\section{Existence of minimal models}

The paper [5] proved that minimal models exist for a $\mathbf{Q}$-factorial DLT pairs $(X, B)$ in the case where the boundary divisor $B$ is big with respect to the pair $(X, B)$ over the base in the following sense:

Definition 6.1. Let $(X, B)$ be an LC pair. A subvariety $Z$ of $X$ is said to be an $L C$ center of $(X, B)$ if there exists a log resolution $\mu: Y \rightarrow$ $(X, B)$ such that, when one writes $\mu^{*}\left(K_{X}+B\right)=K_{Y}+B_{Y}$, there exists an irreducible component $E$ of $B_{Y}$ whose coefficient is equal to one and such that $\mu(E)=Z$.

If $(X, B)$ is a DLT pair, then it is easy to see that $Z$ is an LC center if and only if it is an irreducible component of the intersection of some of the irreducible components of $B$ whose coefficients are equal to one.

Definition 6.2. Let $f:(X, B) \rightarrow T$ be a projective morphism from a DLT pair. An R-Cartier divisor $D$ on $X$ is said to be big with respect to $(X, B)$ if one can write $D=A+E$ for an ample $\mathbf{R}$-Cartier divisor $A$ and an effective R-Cartier divisor $E$ whose support does not contain any LC center of $(X, B)$.

$D$ is called pseudo-effective with respect to $(X, B)$ if $D+A$ is big with respect to $(X, B)$ for any ample $\mathbf{R}$-Cartier divisor $A$.

There are many advantages to consider big boundaries. If $B$ is big with respect to $(X, B)$, then the following hold:

- The $\log$ canonical divisor $K_{X}+B$ is semi-ample, i.e., it is numerically equivalent to a pull-back of an ample $\mathbf{R}$-Cartier divisor by a morphism. In other words, the Abundance Conjecture holds in this case. In particular, if $B$ is a $\mathbf{Q}$-divisor, then there exists a positive integer $m$ such that $m\left(K_{X}+B\right)$ is relatively base point free over the base. This is a consequence of the Base Point Free Theorem.

- The number of faces on the nef cone which was considered in the MMP is finite even if $\epsilon$ goes to zero. This is a consequence of the Cone Theorem.

- The number of marked minimal models for a fixed pair is finite, where a marked minimal model is a minimal model together with a birational map from the original pair. This is one of the results proved in [5].

We note that there are examples where items 2 and 3 are false when $B$ is not big.

Hacon and McKernan [14] proved the following existence theorem of the flip (when combined with results in $[\mathbf{5}]$ ):

Theorem 6.3 (Flip Theorem). Let $(X, B)$ be a Q-factorial DLT pair which is projective over a base, and $\phi: X \rightarrow Y$ a small contraction morphism in the MMP for $K_{X}+B$. Then there exists a flip $\phi^{\prime}: X^{\prime} \rightarrow Y$. 
In order to prove the Flip Theorem, it is sufficient to consider the case where the pair $(X, B)$ is KLT and $B$ is a $\mathbf{Q}$-divisor by perturbing the coefficients of $B$ because the ampleness is an open condition. In this case, the existence of a flip is equivalent to the finite generatedness of the sheaf of graded $\mathcal{O}_{Y}$-algebras

$$
\bigoplus_{m=0}^{\infty} \phi_{*} \mathcal{O}_{X}\left(\left\llcorner m\left(K_{X}+B\right)\right\lrcorner\right)
$$

over $\mathcal{O}_{Y}$, which is a a special case of the Finite Generation Theorem. In other words, the inductive procedure of the MMP decomposes a difficult global finite generation problem into easier local finite generation problems.

The termination conjecture of flips has not yet been fully proved. But [5] proved a partial termination theorem for directed flips with scaling:

Theorem 6.4 (Scaled Termination Theorem). Let $f:(X, B) \rightarrow T$ be a projective morphism from a $\mathbf{Q}$-factorial DLT pair to a quasi-projective variety. Assume that $B$ is big over $T$ with respect to $(X, B)$. Then the MMP for $(X, B)$ with scaling always terminates after a finite number of steps.

By the MMP, we have the following consequence:

TheOREM 6.5 (Existence of Models). Let $f:(X, B) \rightarrow T$ be a projective morphism from a Q-factorial DLT pair to a quasi-projective variety. Assume that $B$ is big over $T$ with respect to $(X, B)$. Then there exists is a birational map $\alpha:(X, B) \rightarrow\left(X^{\prime}, B^{\prime}\right)$ over $T$ to a $\mathbf{Q}$-factorial DLT pair with a projective morphism $f^{\prime}: X^{\prime} \rightarrow T$ which satisfies the following conditions:

(1) $\alpha$ is surjective in codimension one, and $B^{\prime}=\alpha_{*} B$.

(2) If $\mu: Y \rightarrow X$ and $\mu^{\prime}: Y \rightarrow X^{\prime}$ are common resolutions, then $\mu^{*}\left(K_{X}+B\right)-\left(\mu^{\prime}\right)^{*}\left(K_{X^{\prime}}+B^{\prime}\right)$ is an effective $\mathbf{R}$-divisor whose support contains strict transforms of all the exceptional prime divisors of $\alpha$.

(3) If $\left[K_{X}+B\right] \in \operatorname{Psef}(X / T)$, then $\left(X^{\prime}, B^{\prime}\right)$ is a minimal model of $(X, B)$ over $T$, i.e., $K_{X^{\prime}}+B^{\prime}$ is $f^{\prime}$-nef. On the other hand, if $\left[K_{X}+B\right] \notin P \operatorname{sef}(X / T)$, then $\left(X^{\prime}, B^{\prime}\right)$ has a Mori fiber space structure $\phi: X^{\prime} \rightarrow Y$ over $T$, where $\operatorname{dim} Y<\operatorname{dim} X^{\prime}$ and $-\left(K_{X^{\prime}}+B^{\prime}\right)$ is $\phi$-ample.

Now we state the Finite Generation Theorem. We note that we do not need to assume the bigness of the boundary:

TheOrem 6.6 (Finite Generation Theorem). Let $f:(X, B) \rightarrow T$ be a projective morphism from a KLT pair to a quasi-projective variety. Assume in addition that the boundary $B$ is a $\mathbf{Q}$-divisor. Then the relative canonical ring

$$
R\left(X / T, K_{X}+B\right)=\bigoplus_{m=0}^{\infty} f_{*} \mathcal{O}_{X}\left(\left\llcorner m\left(K_{X}+B\right)\right\lrcorner\right)
$$

is finitely generated as a graded $\mathcal{O}_{T}$-algebra. 
We note that the assumption of $B$ being a $\mathbf{Q}$-divisor is indispensable. Indeed the statement is clearly false for a non-rational R-divisor. On the other hand, the limiting argument in the proof of the existence of minimal models makes it necessary to consider $\mathbf{R}$-divisors in the proof.

The proof of the Finite Generation Theorem is as follows. If $B$ is big with respect to the pair, then the theorem follows from the Base Point Free theorem together with the existence theorem of a minimal model. For the general case, we use the following reduction theorem $([\mathbf{1 2}])$ :

TheOREm 6.7. Let $f:(X, B) \rightarrow T$ be a projective morphism from a KLT pair to a quasi-projective variety. Assume that the boundary $B$ is a Q-divisor, and that $\kappa\left(X_{\eta},\left.\left(K_{X}+B\right)\right|_{X_{\eta}}\right) \geq 0$ for the generic fiber $X_{\eta}$ of $f$. Then there exists a projective birational morphism $\mu: Y \rightarrow X$ from a smooth variety, a surjective morphism $f: Y \rightarrow Z$ to a smooth variety projective over $T$, a $\mathbf{Q}$-divisor $C=\sum c_{j} C_{j}$ on $Z$ such that $\sum C_{j}$ is a normal crossing divisor and $c_{i} \in(0,1)$, and a $\mathbf{Q}$-divisor $L$ on $Z$ which is nef over $T$ such that the following are satisfied:

(1) $K_{Z}+C+L$ is big over $T$.

(2) $f \circ \mu^{-1}$ induces an isomorphism of graded $\mathcal{O}_{T}$-algebras:

$$
R\left(X / T, K_{X}+B\right) \cong R\left(Z / T, K_{Z}+C+L\right) .
$$

The outline of the proof of this theorem is as follows. We consider a rational map, called the Iitaka fibration, defined by some positive multiple of $K_{X}+B$ whose image has relative dimension equal to $\kappa\left(X_{\eta},\left.\left(K_{X}+B\right)\right|_{X_{\eta}}\right)$ over $T$, and modify it by changing birational models and using covering tricks. Then we apply the semi-positivity theorem of the Hodge bundles in $[\mathbf{1 9}]$.

The rest of the proof of the Finite Generation Theorem is as follows. We write $K_{Z}+C+L=A+E$ for an ample Q-divisor $A$ and an effective Q-divisor $E$. Then we apply the big case to a new pair $(1+\epsilon)\left(K_{Z}+C+L\right)=$ $K_{Z}+(C+\epsilon E)+(L+\epsilon A)$ for a small positive rational number $\epsilon$.

The following Polytope Decomposition Theorem for varying boundaries was proved in [5] after Shokurov [52]. This theorem is interesting in its own right besides its importance for the termination argument. We formulate the theorem in a more exact form and in the case where the boundary is not necessarily big. The first part is a decomposition according to the canonical models:

Theorem 6.8 (Polytope Decomposition Theorem 1). Let $(X, \bar{B})$ be a Q-factorial KLT pair with a projective morphism $f: X \rightarrow T$ to a base space, $B_{1}, \ldots, B_{r}$ effective $\mathbf{Q}$-Cartier divisors, and $\bar{V}$ a polytope contained in the set $\left\{B=\sum_{i} b_{i} B_{i} \mid b_{i} \in \mathbf{R}\right\} \cong \mathbf{R}^{r}$ such that the pairs $(X, B)$ are $L C$ for all $B \in \bar{V}$. Consider a closed convex subset

$$
V=\left\{B \in \bar{V} \mid\left[K_{X}+B\right] \in \operatorname{Psef}(X / T)\right\} .
$$

Assume that for each $B \in V$, there exist a minimal model $\alpha:(X, B) \rightarrow-\rightarrow$ $(Y, C)$ and a canonical model $g: Y \rightarrow Z$ for $f:(X, B) \rightarrow T$. Moreover 
assume that there exists a real number $\epsilon>0$ for each $B$ such that the morphism $g:\left(Y, \alpha_{*} B^{\prime}\right) \rightarrow Z$ for $B^{\prime} \in \bar{V}$ has minimal and canonical models whenever $B^{\prime} \in P \operatorname{sef}(Y / Z)$ and $\left\|B^{\prime}-B\right\| \leq \epsilon$, where \|\| denotes the maximum norm of the coefficients. Then there exists a finite decomposition to disjoint subsets

$$
V=\coprod_{j=1}^{s} V_{j}
$$

with rational maps $\beta_{j}: X \rightarrow Z_{j}$ which satisfies the following conditions:

(1) $B \in V_{j}$ if and only if $\beta_{j}$ gives the canonical model for $f:(X, B)$ $\rightarrow T$.

(2) The closures $\bar{V}_{j}$, hence $V$, are polytopes for all $j$. Moreover, if $\bar{V}$ is a rational polytope, then so are the $\bar{V}_{j}$ and $V$.

The second part is a finer decomposition according to the minimal models:

TheOREM 6.9 (Polytope Decomposition Theorem 2). Under the same assumptions as the above theorem, each $V_{j}$ are further decomposed into a finite disjoint union

$$
V_{j}=\coprod_{k=1}^{t} W_{j, k}
$$

which satisfies the following conditions: let $\alpha: X \rightarrow Y$ be a birational map such that

$$
W=\{B \in V \mid \alpha \text { is a minimal model for }(X, B)\}
$$

is non-empty. Then

(1) There exists an index $j$ such that $W \subset \bar{V}_{j}$.

(2) If $W \cap V_{j}$ is non-empty for some $j$, then $W \cap V_{j}$ coincides with one of the $W_{j, k}$.

(3) The closure $\bar{W}_{j, k}$ is a polytope for any $j$ and $k$. Moreover, if $\bar{V}$ is a rational polytope, then so are the $\bar{W}_{j, k}$.

The existence of $\bar{B}$ is assumed only to ensure that the MMP works well. In the case where the $B \in V$ are big with respect to the pairs, the existence of minimal and canonical models is already proved, and the conditions in the above theorems are always satisfied.

We note that the conclusions of the above theorems do not contradict with examples in [25], where there are infinitely many chambers, even if the boundaries are not big. The reason is that our finite decomposition theorem is a statement of local nature in a sense.

The termination of the flips for the scaled MMP is an easy consequence of the Polytope Decomposition Theorem. Indeed the models before and after a flip correspond to different chambers. There are only finitely many chambers on the line segment on which the scaled MMP is played, hence the termination. 


\section{The vanishing theorems and the extension techniques}

Now we start to explain some ideas of proofs of the theorems. The vanishing theorems of Kodaira type have surprizingly diverse applications in the birational geometry. We need to assume that the characteristic of the base field is zero.

We start with the original Kodaira vanishing theorem ([31]):

Theorem 7.1 (Kodaira Vanishing Theorem). Let $X$ be a smooth projective variety, and $D$ a divisor. If $D-K_{X}$ is ample, then $H^{p}(X, D)=0$ for $p>0$.

By applying a covering trick to the Kodaira vanishing theorem, we deduce its $\log$ and relative version $([\mathbf{2 0}, \mathbf{6 2}, \mathbf{2 9}])$ :

Theorem 7.2 (Kawamata-Viehweg Vanishing Theorem). Let $X$ be a normal variety, $f: X \rightarrow T$ a projective morphism to a base, $B$ an effective R-divisor, and $D$ a Cartier divisor. If $(X, B)$ is $K L T$ and $D-\left(K_{X}+B\right)$ is $f$-ample, then $R^{p} f_{*} \mathcal{O}_{X}(D)=0$ for $p>0$.

This generalization is used as in the following way. Let $D$ be a divisor on a smooth projective variety $X$. Suppose that $D-K_{X}$ is not ample, but close to ample. We can sometimes find a small effective $\mathbf{R}$-divisor $B$ by a perturbation such that $(X, B)$ is KLT and $D-\left(K_{X}+B\right)$ is ample. Then we have still the vanishing $H^{p}(X, D)=0$ for $p>0$.

The Cone and Contraction Theorems are proved by using the above Vanishing Theorem ([29]). The method of proofs are called $x$-method, which is a cohomological technique for proving base point freeness using the vanishing theorem. The name came from the surprizingly similar applications of the vanishing theorem toward apparently different first two problems in Reid's list ([48]). The same method was later applied toward Fujita's conjecture on the base point freeness of adjoint systems.

The idea of the proof of the base point free theorem is as follows. The point is to extend a holomorphic section from a codimension one subvariety to the whole space, and use the induction on the dimension. Suppose that we want to prove the base point freeness of a complete linear system $|m D|$ on a smooth projective variety $X$ for a large integer $m$. First we take a positive integer $m_{1}$ such that $\left|m_{1} D\right|$ is non-empty, where the existence of such an $m_{1}$ is guaranteed by the Non-Vanishing Theorem which is also proved by the $x$-method. We want to make the base locus smaller by taking a larger integer $m_{2}$ so that the base locus disappears eventually. We construct a projective birational morphism $\mu: Y \rightarrow X$ from a smooth variety with an effective divisor $E$ and a smooth prime divisor $Z$ such that:

(1) The support of $E$ is contracted by $\mu$.

(2) The image of $Z$ by $\mu$ is contained in the base locus of $\left|m_{1} D\right|$.

The argument for finding such a situation looked tricky when it was found, but we now know that the log canonical threshold is the concept 
hidden in this argument. We consider an exact sequence

$$
\begin{aligned}
0 & \rightarrow \mathcal{O}_{Y}\left(m_{2} \mu^{*} D+E-Z\right) \rightarrow \mathcal{O}_{Y}\left(m_{2} \mu^{*} D+E\right) \\
& \rightarrow \mathcal{O}_{Z}\left(\left.\left(m_{2} \mu^{*} D+E\right)\right|_{Z}\right) \rightarrow 0
\end{aligned}
$$

for a multiple $m_{2}$ of $m_{1}$. If $m_{2}$ is suitably large, then we prove that

$$
H^{0}\left(Z,\left.\left(m_{2} \mu^{*} D+E\right)\right|_{Z}\right) \neq 0
$$

by using the Non-Vanishing Theorem, and

$$
H^{1}\left(Y, m_{2} \mu^{*} D+E-Z\right)=0
$$

by using the Vanishing Theorem. By condition 1, the natural homomorphism

$$
\mu_{*}: H^{0}\left(Y, m_{2} \mu^{*} D+E\right) \rightarrow H^{0}\left(X, m_{2} D\right)
$$

is bijective. It follows that $\mu(Z)$ is not contained in the base locus of $\left|m_{2} D\right|$, hence it is strictly smaller that that of $\left|m_{1} D\right|$.

The proof of the Non-Vanishing Theorem is similar, but we take an artificial non-complete linear system compared to the natural one $|m D|$. The proof of the Cone Theorem is also similar.

We worked "upstairs" in the above proof, i.e., on a resolution which lies above the original variety, and extend holomorphic sections from a divisor to the whole variety by using the Vanishing Theorem for line bundles. If we use the vanishing theorem for multiplier ideal sheaves explained below, we can work "downstairs", i.e., on the original variety, for the extension argument. Then we can obtain more powerful extension theorems because it becomes possible to consider an infinite series of linear systems simultaneously.

We extend the vanishing theoren to the case where a pair $(X, B)$ consisting of a normal variety and an effective $\mathbf{R}$-divisor such that $K_{X}+B$ is an $\mathbf{R}$-Cartier divisor but the pair is not necessarily KLT.

Definition 7.3. Let $(X, B)$ be a pair consisting of a normal variety and an effective $\mathbf{R}$-divisor such that $K_{X}+B$ is an $\mathbf{R}$-Cartier divisor. Let $\mu$ : $Y \rightarrow X$ be a log resolution and we write $\mu^{*}\left(K_{X}+B\right)=K_{Y}+\sum e_{j} E_{j}$, where $\sum E_{j}$ is a normal crossing divisor. The multiplier ideal sheaf $I(X, B) \subset \mathcal{O}_{X}$ is defined by the following formula

$$
I(X, B)=\mu_{*} \mathcal{O}_{Y}\left(-\sum\left\llcorner e_{j}\right\lrcorner E_{j}\right) .
$$

It is independent of the choice of the log resolution. We have $I(X, B)=$ $\mathcal{O}_{X}$ if and only if $e_{j}<1$ for all $j$, i.e., if $(X, B)$ is KLT.

Let $f: X \rightarrow T$ be a projective morphism and $D$ a Cartier divisor on $X$. If $D-\left(K_{X}+B\right)$ is $f$-ample, then Theorem 7.2 implies that

$$
R^{p} \mu_{*} \mathcal{O}_{X}\left(\mu^{*} D-\sum\left\llcorner e_{j}\right\lrcorner E_{j}\right)=0
$$


and

$$
R^{p}(f \circ \mu)_{*} \mathcal{O}_{X}\left(\mu^{*} D-\sum\left\llcorner e_{j}\right\lrcorner E_{j}\right)=0
$$

for $p>0$. Hence we have a vanishing theorem for non-KLT pairs:

TheOREM 7.4 (Nadel Vanishing Theorem). Let $f: X \rightarrow T$ be a projective morphism from a normal variety, $B$ an effective $\mathbf{R}$-divisor such that $K_{X}+B$ is an $\mathbf{R}$-Cartier divisor, and $D$ a Cartier divisor. If $D-\left(K_{X}+B\right)$ is $f$-ample, then $R^{p} f_{*}\left(I(X, B) \mathcal{O}_{X}(D)\right)=0$ for $p>0$.

The above theorem was first proved by Nadel [42] in a more general complex analytic setting (but on a smooth underlying variety). Indeed the multiplier ideal sheaf is defined for a line bundle $L$ with a singular hermitian metric on a complex manifold $X$. A singular hermitian metric $h$ is a degenerate hermitian metric which can be written locally as $h=h_{0} e^{-\phi}$, where $h_{0}$ is a $C^{\infty}$ metric and $\phi$ is a locally integrable weight function. The multiplier ideal sheaf $I(L, h)$ is defined as the largest ideal sheaf such that all local sections of $I(L, h) L$ satisfy locally the $L^{2}$ condition with respect to the metric. It is proved to be a coherent sheaf on $X$.

For example, assume that $X$ is an open subset of $\mathbf{C}^{n}$ and $B=\sum b_{i} B_{i}$ is an effective $\mathbf{R}$-divisor. If the prime divisors $B_{i}$ have local equations $g_{i}=0$, then we can define an algebraically defined singular hermitian metric on a trivial bundle $L$ by using the weight function $\phi=\sum_{i} b_{i} \log \left|g_{i}\right|$. In this case, the algebraic and analytic multiplier ideal sheaves coincide: $I(X, B)=I(L, h)$.

The point is that there are a lot more metrics which are essentially different from algebraic metrics. One can use a limit of a sequence of algebraic metrics to produce a non-algebraic one provided that we can prove certain convergence. For example, the analytic Zariski decomposition $([\mathbf{6 0}, \mathbf{9}])$ is a singular hermitian metric which is defined naturally for an arbitrary pseudoeffective line bundle. The metric on a Hodge bundle is another example of analytic metrics.

A new extension technique using the multiplier sheaves was developed by Siu [55] when he proved the deformation invariance of plurigenera:

THEOREM 7.5. Let $f: X \rightarrow T$ be a smooth projective morphism. Then the plurigenus $\operatorname{dim} H^{0}\left(X_{t}, m K_{X_{t}}\right)$ of a fiber $X_{t}=f^{-1}(t)$ is independent of $t \in T$ for any positive integer $m$.

Nakayama [43] proved that positive solutions for conjectures in the MMP including the Abundance Conjecture imply the invariance of plurigenera. But Siu proved the theorem in one step without using an inductive approach of the MMP. The theorem is proved in the case where a fiber $X_{t}$ is of general type in [55], and in general in [56] (see also [59] and [47]). The vanishing theorem used in these proofs is the Ohsawa-Takegoshi type extension theorem $([\mathbf{4 5}, \mathbf{5 5}])$ :

Theorem 7.6 (Ohsawa-Takegoshi Extension Theorem). Let X be a Stein manifold, and $Y$ a smooth hypersurface defined by a bounded holomorphic 
function $t$. Let $(L, h)$ be a line bundle on $X$ with a singular hermitian metric, and $s \in H^{0}\left(Y, K_{Y}+\left.L\right|_{Y}\right)$ a holomorphic section. Assume that the curvature current $-d d^{c} \log h$ is semipositive as a real current of type $(1,1)$, and that $\int_{Y}|s|^{2} h<\infty$. Then there exists an extension $\tilde{s} \in H^{0}\left(X, K_{X}+L\right)$ such that $\tilde{s}=s \wedge d t$ on $Y$ and

$$
\int_{X}|\tilde{s}|^{2} h \leq C \sup |t|^{2} \int_{Y}|s|^{2} h
$$

for a universal constant $C$.

The advantage of Theorem 7.6 is that we do not need to assume the strict positivity of the metric, which corresponds roughly to the ampleness or bigness. But the semipositivity for the metric is strictly stronger the corresponding algebraic concept of the nefness. This is the reason why there is still no algebraic proof for the invariance theorem of plurigenera.

The idea of the proof of the invariance of plurigenera in the case where the fibers are of general type is to use a construction downstairs which is similar to the Zariski decomposition. The spaces of sections of pluricanonical systems on the central fiber define a series of metrics on the canonical line bundle, hence a sequence of multiplier ideal sheaves. Similar constructions on the total space of the deformations define different metrics, hence a different sequence of multiplier ideal sheaves. A suitable vanishing theorem relates these ideals, and the extension of sections is proved.

This proof allowed an algebraic analogue in [26] (see also [44] and [27]) because the canonical line bundle is big in this case. The algebraic version of the extension theorem were generalized to the logarithmic situation in [13] and [58] (see also [61]). The logarithmic extension theorem was used in the proof of the PL flip theorem [14] explained later. We note that the algebraization of the proof in the general case, i.e., non general type case, is still an open problem.

Now we state the logarithmic extension theorem:

THEOREM 7.7. Let $f: X \rightarrow T$ be a projective morphism from a smooth variety to an affine variety, and $B=\sum b_{i} B_{i}$ a $\mathbf{Q}$-divisor whose support is a normal crossing divisor and such that only one coefficient $b_{0}$ is equal to 1 and other coefficients satisfy $b_{i} \in(0,1)$ for $i \neq 0$. Set $Y=B_{0}$. Let $r$ be a positive integer such that $r\left(K_{X}+B\right)$ has integral coefficients. Set $\left.\left(K_{X}+B\right)\right|_{Y}=K_{Y}+B_{Y}$. Assume the following conditions:

(1) $K_{X}+B$ is pseudo-effective with respect to $(X,\ulcorner B\urcorner)$.

(2) $B-Y$ is big with respect to $(X, Y)$.

Then natural homomorphisms

$$
H^{0}\left(X, m r\left(K_{X}+B\right)\right) \rightarrow H^{0}\left(Y, m r\left(K_{Y}+B_{Y}\right)\right)
$$

are surjective for all positive integers $m$.

This is a correct log generalization of the extension theorem, while [27] Example 4.3 showed that a naive extension is false. 


\section{Flip theorem}

We start to explain how the remaining two conjectures on the flips, the existence and the termination, are proved for the scaled MMP under the additional assumption that the boundary is big.

The basic idea is to use the induction on the dimensions using the adjunction formula which relates canonical divisors in different dimensions: if $Y$ is a smooth divisor on a smooth variety $X$, then we have

$$
\left.\left(K_{X}+Y\right)\right|_{Y}=K_{Y}
$$

This is a very different approach from the proof in dimension three in $[\mathbf{3 9}]$.

We need two theorems, the Special Termination Theorem and the reduction to PL flips, due to Shokurov [53] (see also [11]) preceding to the proof of the flip theorem. The first one is on the termination of flips along boundary components with coefficient 1 :

TheOrem 8.1 (Special Termination). Let $(X, B)$ be a $\mathbf{Q}$-factorial DLT pair of dimension $n$ which is projective over a base. Assume that the MMP holds, i.e., the existence and termination conjectures hold, in dimension less than $n$. Let

$$
(X, B)=\left(X_{0}, B_{0}\right) \rightarrow\left(X_{1}, B_{1}\right) \rightarrow-\rightarrow
$$

be an infinite sequence of flips. Then there exists a positive integer $m_{0}$ such that the flip $\left(X_{m}, B_{m}\right) \rightarrow\left(X_{m+1}, B_{m+1}\right)$ is an isomorphism in a neighborhood of $\left\llcorner B_{m}\right\lrcorner$ for every $m \geq m_{0}$.

Similarly, if the MMP holds in dimension less than $n$ under the additional condition that the MMP is scaled or the boundary is big, then the special termination holds under the additional assumption that the MMP is scaled or the boundary $B$ is big with respect to $(X, B)$.

The idea of the proof is to use the adjunction formula to reduced boundary components. It turned out that the Special Termination Theorem, not the general termination, is sufficient to prove the existence of minimal models thanks to the Non-Vanishing Thoerem explained in the next section.

A small contraction morphism of a $\mathbf{Q}$-factorial DLT pair $\phi:(X, B) \rightarrow Y$ in the MMP is said to be a $P L$ (prelimiting) contraction, if there is an irreducible component $S$ of $\llcorner B\lrcorner$ such that $-S$ is $\phi$-ample. A flip for a $\mathrm{PL}$ contraction is called a $P L$ flip. The second one is the reduction to PL flips:

THEOREM 8.2. Assume that the special termination theorem for the scaled MMP with big boundaries and the existence of the PL flip hold in dimension $n$. Then the existence of the flip in general holds in the same dimension.

The idea of the proof is to introduce additional artificial boundary as a scaling, and then reduce the boundary back to the original state gradually by using the scaled MMP. This is the first appearance of the MMP with scaling. 
Hacon and McKernan [14] proved the existence of a PL flip:

THEOREM 8.3. Assume that a minimal models exists for any KLT pair of dimension $n-1$ when the boundary is big. Then a flip exists for arbitrary $P L$ small contraction in dimension $n$.

The idea of proof is as follows. Let $\phi:(X, B) \rightarrow Y$ be a PL contraction morphism. We may assume that $B$ is a $\mathbf{Q}$-divisor. Let $S$ be an irreducible component of $\llcorner B\lrcorner$ such that $-S$ is $\phi$-ample. We use the adjunction formula: $\left.\left(K_{X}+B\right)\right|_{S}=K_{S}+B_{S}$, where $B_{S}$ is usually larger than the restriction $\left.B\right|_{S}$ because of the singularities of $X$, namely the subadjunction. In order to prove the finite generation of the relative canonical ring

$$
R\left(X / Y, K_{X}+B\right)=\bigoplus_{m=0}^{\infty} \phi_{*} \mathcal{O}_{X}\left(\left\llcorner m\left(K_{X}+B\right)\right\lrcorner\right)
$$

it is sufficient to prove the finite generation of the image of the restriction homomorphism

$$
R\left(X / Y, K_{X}+B\right) \rightarrow R\left(S / Y, K_{S}+B_{S}\right) .
$$

The target of this ring homomorphism is finitely generated by the induction hypothesis, but the difficulty is that the homomorphism is not surjective. The point of the proof of the PL flip theorem is to identify the image of this homomorphism.

By restricting $m$-canonical systems on $X$ to $S$ and cancelling fixed components from the boundary $B_{S}$, we obtain a series of boundaries $B_{S^{\prime}, m}$ depending on $m$ on a fixed birational model $S^{\prime}$ of $S$. By using the extension theorem (Theorem 7.7), we prove that all the pluricanonical forms with respect the boundaries $B_{S^{\prime}, m}$ on $S^{\prime}$ are in the images of the restriction homomorphisms. By the Polytope Decomposition Theorem in dimension $n-1$, we find a fixed birational model $S^{\prime \prime}$ of $S$ which dominates all the minimal models corresponding to this series of boundary divisors. Then we prove that certain stability of the movable parts of $m$-canonical systems holds when $m$ goes to infinity by using the vanishing theorem and the effective version of the Base Point Free Theorem by Kollár [34].

We note that arbitrary divisor is big for a birational map such as $\phi$. The difficulty concerning the infinity arising from the fact that we should consider all the $m$-canonical systems at the same time is solved by the finiteness statement of the Polytope Decomposition Theorem.

\section{Termination of flips and the non-vanishing theorem}

An old approach to the termination conjecture is to use invariants of singularities initiated by Shokurov [50]. Let us consider the simplest case; let $X$ be a three dimensional variety with only terminal singulsrities, and $\mu: Y \rightarrow X$ a resolution of singularities. We write $\mu^{*} K_{X}=K_{Y}+\sum e_{j} E_{j}$. By the assumption, we have $e_{j}<0$ for all $j$. The difficulty of the variety $X$ 
is the number of $j$ such that $-1<e_{j}$. One can prove that the difficulty is a well defined non-negative integer, and it decreases strictly after a flip. The termination follows immediately.

If the singularity is worse or if the dimension is higher, then similar properties, the well-definedness and the monotoneness, fail. But we can modify the definition of the difficulty and obtain some termination theorems. The following is the known results up to now obtained by using the concept of difficulty:

THEOREM 9.1. (1) $([\mathbf{2 3}, \mathbf{5 2}])$ The termination conjecture holds for arbitrary DLT pairs in dimension three.

(2) ([1]) The termination conjecture holds for a $K L T$ pair $(X, B)$ of $d i$ mension four if $-\left(K_{X}+B\right)$ is numerically equivalent to an effective $\mathbf{R}$-divisor over the base.

(3) ([1]) If $\left(X, \sum b_{i} B_{i}\right)$ is a KLT pair of dimension four such that $c_{0} K_{X}+\sum c_{i} B_{i}$ is relatively big over the base for some numbers $c_{i} \in \mathbf{R}$. Then there exists a process of the MMP with scaling which terminates.

In particular, the existence of a minimal model for arbitrary DLT pair $(X, B)$ over a base is proved only in the case $\operatorname{dim} X=3$ at the moment.

Birkar, Cascini, Hacon and McKernan [5] took a very different approach to the termination conjecture. They did not prove the termination as an isolated statement, but rather included it into a chain of statements concerning the MMP. By using the induction on dimension, the termination is reduced to the special termination as in the following way.

Let $(X, B)$ be a DLT pair which is projective over a base $T$. Assume that the $\log$ canonical divisor $K_{X}+B$ is numerically equivalent to an effective $\mathbf{R}$-divisor $M$ over $T$. Then the minimality question for $(X, B)$ is equivalent to that for $(X, B+t M)$ for $t>0$ as long as the the new pair is LC. Assume for simplicity that $(X, B+t M)$ is DLT for some $t>0$ and that the support of $\llcorner B+t M\lrcorner$ coincides with that of $M$. By applying the Special Termination Theorem, we conclude that the flips terminates near $\llcorner B+t M\lrcorner$. Since $M$ is numerically equivalent to $K_{X}+B$, it follows that $K_{X}+B$ becomes nef in this process. In the general case, we need more careful argument on the support of $M$ during the process of the MMP with scaling.

There is a modified version of the above termination argument in Birkar [4]. In particular it is proved that, if $K_{X}+B$ is numerically equivalent to an effective $\mathbf{R}$-divisor, then a minimal model exists if $\operatorname{dim} X \leq 5$.

The remaining problem is to prove the existence of an effective $\mathbf{R}$-divisor $M$. This is a generalization of the Non-Vanishing Theorem:

TheOREM 9.2. Let $(X, B)$ be a KLT pair which is projective over a base $T$. Assume the following conditions:

(1) $K_{X}+B$ is pseudo-effective over $T$.

(2) $B$ is big over $T$. 
Then there exists an effective $\mathbf{R}$-divisor $M$ which is numerically equivalent to $K_{X}+B$ over $T$.

The assertion is only a part of the existence theorem of a minimal model, but this is the key point in its proof. The proof in [5] is also a generalization of the Non-Vanishing Theorem which is a part of the Base Point Free Theorem which is already proved when $K_{X}+B$ is nef, together with the idea similar to the proof of the flip theorem. We use the minimal model theorem which is already proved under the additional assumption that $K_{X}+B$ is effective, and the Polytope Decomposition Theorem in dimension one less.

The proof proceeds roughly as follows. As in the case of the NonVanishing Thorem or the reduction theorem to the PL flip, we increase the boundary artificially so that the pair $(X, B)$ becomes DLT and $\llcorner B\lrcorner=Z$ is irreducible. We consider the case where $B$ is a $\mathbf{Q}$-divisor for simplicity. If we add an ample $\mathbf{Q}$-divisor $\epsilon H$ to the boundary, then $K_{X}+B+\epsilon H$ becomes big, and there exists a minimal model for the pair $(X, B+\epsilon H)$. If we take the limit $\epsilon \rightarrow 0$, then there may be infinitely many chambers in the space of divisors corresponding to the minimal models of the pairs $(X, B+\epsilon H)$. By using the Special Termination Theorem, we can prove that certain neighborhood of $Z$ becomes stable under this infinite chain of wall crossings. Then we can fix a minimal model of $Z$, on which there exists a desired section thanks to the usual Base Point Free Theorem. By using the Vanishing Theorem, we infer that this section is extended to a birational model of $X$.

The assumption that the boundary $B$ is big is indispensable in the first step of the above proof where we find $Z$. Indeed as is shown in [4], the Non-Vanishing Thorem is the most difficult point if we try to extend the minimal model theory to the case where the boundary is not necessarily big.

\section{Birational maps between birational models}

The output of the MMP, a minimal model or a Mori fiber space, is not uniquely determined in general when we start with a fixed pair, because there are choices of the faces in the process of the MMP. We would like to control the non-uniqueness. The answer is given by the Polytope Decomposition Theorem again.

The non-uniqueness of a minimal model for a fixed variety is a new phenomena in dimension three or higher. The following theorem asserts that the existence of flops is the only reason.

A flop for a pair $(X, B)$ is a diagram

$$
X \stackrel{\phi}{\longrightarrow} Y \stackrel{\phi^{+}}{\longleftarrow} X^{+}
$$

which is a flip for another pair $\left(X, B^{\prime}\right)$, where $B^{\prime}$ is a suitably chosen different boundary, and such that $K_{X}+B$ is numerically trivial for $\phi ;\left[K_{X}+B\right]=0$ in $N^{1}(X / Y)$.

TheOREm 10.1. Let $f:(X, B) \rightarrow T$ be a projective morphism from a $K L T$ pair, let $g:(Y, C) \rightarrow T$ and $g^{\prime}:\left(Y^{\prime}, C^{\prime}\right) \rightarrow T$ be its minimal models, 
and let $\alpha: Y \rightarrow Y^{\prime}$ be the induced birational map over $T$. Assume that there exists a canonical model of $(X, B)$. Then $\alpha$ is decomposed into a sequence of flops in the follwoing way: there exists an effective $\mathbf{Q}$-Cartier divisor $D$ on $Y$ such that $(Y, C+D)$ is still KLT and such that $\alpha$ becomes a composition of a sequence of birational maps

$$
Y=Y_{0} \rightarrow Y_{1} \rightarrow \cdots-\rightarrow Y_{l}=Y^{\prime}
$$

such that $\alpha_{k}: Y_{k-1} \rightarrow Y_{k}(1 \leq k \leq l)$ is a flop for the pair $\left(Y_{k-1}, C_{k-1}\right)$ as well as a flip for the pair $\left(Y_{k-1}, C_{k-1}+D_{k-1}\right)$, where $C_{k-1}$ and $D_{k-1}$ are the strict transforms of $C$ and $D$, respectively.

We note that $\alpha$ is an isomorphism in codimension one as we already know. We remark that the boundary $B$ need not to be big. There is a differnt version of the factorization theorem in [28], where we do not need to assume the existence of a canonical model, but we have to assume that $B$ is a $\mathbf{Q}$-divisor.

A marked minimal model is a pair consisting of a minimal model and a birational map to a fixed reference model. The number of birationally equivalent marked minimal models is finite if the boundary $B$ is big, but it is not the case in general $([\mathbf{2 5}])$. The above theorem claims that there are still only finitely many marked minimal models which lie between two different minimal models. It is conjectured that the number of birationally equivalent minimal models is finite up to isomorphisms, i.e., when we forget the markings.

We use the Polytope Decomposition Theorem to prove the above theorem in the following way. We take a general ample $\mathbf{R}$-Cartier divisor $H$ and $H^{\prime}$ on $Y$ and $Y^{\prime}$, respectively, and we consider a triangle spanned by $C, C+H$ and $C+H^{\prime}$ in the space of divisors on $Y$, where the strict transform of $H^{\prime}$ on $Y$ is denoted by the same letter. The canonical model coresponds to the chamber $\{0\}$. If we choose $H$ and $H^{\prime}$ small enough, then the closures of the chambers $V$ and $V^{\prime}$ corresponding to the models $Y$ and $Y^{\prime}$ contain 0. Moreover the union of the chambers between $V$ and $V^{\prime}$ which contain 0 in the closures contains the line segment joining the points $C+H$ and $C+H^{\prime}$. Then the wall crossing process provides a decomposition of $\alpha$. By induction on the relative Picard numbers, we obtain eventually the decomposition to flops.

As for the Mori fiber spaces, the non-uniqueness phenomenon appears already in dimension two. For example, ruled surfaces $\mathbf{P}\left(\mathcal{O}_{\mathbf{P}^{1}} \oplus \mathcal{O}_{\mathbf{P}^{1}}(d)\right)$ over $\mathbf{P}^{1}$ for different integers $d$ are all birationally equivalent. An elementary transformation of a ruled surface is a combination of a blowing up at a point in a fiber and the blowing down of the strcit transform of the fiber. If $g_{i}: X_{i} \rightarrow C$ for $i=1,2$ are ruled surfaces over the same curve $C$, then they are connected each other by a sequence of elementary transformations. There is a different kind of decompositions; any birational map $\mathbf{P}^{2}-\rightarrow \mathbf{P}^{2}$ is decomposed into linear and quadratic transformations. The latter is further decomposed into point blowings up, point blowings down, and elementary tansformations. 
The Sarkisov program is a higher dimensional generalization. We can decompose any birational map between the total spaces of Mori fiber spaces into elementary links, generalizations of elementary transformations.

Hacon and McKernan [15] proved the following:

TheOREM 10.2. Let $f:(X, B) \rightarrow T$ be a projective morphism from a KLT pair, let $\phi:(Y, C) \rightarrow Z$ and $\phi^{\prime}:\left(Y^{\prime}, C^{\prime}\right) \rightarrow Z^{\prime}$ be Mori fiber spaces obtained by the MMP from a KLT pair $(X, B)$ over $T$, and let $\alpha: Y \rightarrow Y^{\prime}$ be the induced birational map over $T$ :

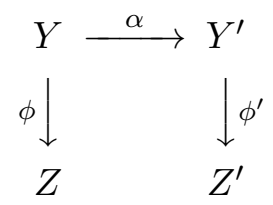

Then there exists a sequence of the following type commutative diagrams called elementary links for $1 \leq k \leq l$ with some positive integer $l$ :

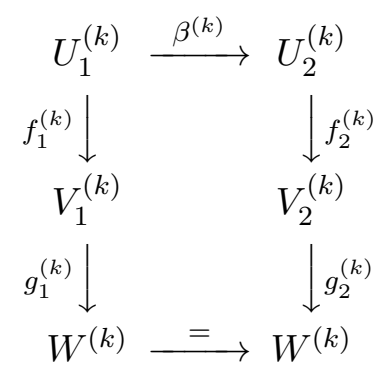

where $\beta^{(k)}$ is a composition of a sequence of flips for a suitably chosen boundary on $U_{1}^{(k)}$ and such that $\alpha$ is decomposed as

$$
\alpha=\alpha_{l} \circ \cdots \circ \alpha_{1}
$$

where each $\alpha_{k}$ is a birational map described in one of the following cases:

(1) $g_{1}^{(k)}$ and $f_{2}^{(k)}$ are Mori fiber spaces, $f_{1}^{(k)}$ is a divisorial contraction, $g_{2}^{(k)}$ is a morphism with relative Picard number one, and $\alpha_{k}=$ $\beta^{(k)} \circ\left(f_{1}^{(k)}\right)^{-1}$.

(2) $g_{1}^{(k)}$ and $g_{2}^{(k)}$ are Mori fiber spaces, $f_{1}^{(k)}$ and $f_{2}^{(k)}$ are divisorial contractions, and $\alpha_{k}=f_{2}^{(k)} \circ \beta^{(k)} \circ\left(f_{1}^{(k)}\right)^{-1}$.

(3) $f_{1}^{(k)}$ and $g_{2}^{(k)}$ are Mori fiber spaces, $f_{2}^{(k)}$ is a divisorial contraction, $g_{1}^{(k)}$ is a morphism with relative Picard number one, and $\alpha_{k}=$ $f_{2}^{(k)} \circ \beta^{(k)}$.

(4) $f_{1}^{(k)}$ and $f_{2}^{(k)}$ are Mori fiber spaces, $g_{1}^{(k)}$ and $g_{2}^{(k)}$ are morphisms with relative Picard number one, and $\alpha_{k}=\beta^{(k)}$.

The theorem was proved in dimension three by Corti [7]. The crucial point of the proof is to prove that a sequence of elementary links terminates. [15] interpreted the sequence of elementary links as a wall crossing process, 
and proved the termination by using the Polytope Decomposition Theorem. It is remarkable that the four types of elementary links which are apparently different have the same interpretation as a wall crossing process in terms of the polytope decomposition.

The argument of [15] is as follows. We take a general ample $\mathbf{R}$-Cartier divisor $H$ (resp. $H^{\prime}$ ) on $Y$ (resp. $Y^{\prime}$ ) such that $K_{Y}+C+H=\phi^{*} L$ (resp. $K_{Y^{\prime}}+C^{\prime}+H^{\prime}=\left(\phi^{\prime}\right)^{*} L^{\prime}$ ) for some ample $\mathbf{R}$-Cartier divisor $L$ (resp. $L^{\prime}$ ) on $Z$ (resp. $Z^{\prime}$ ), and let $D$ (resp. $D^{\prime}$ ) be the strict transforms of $H$ (resp. $H^{\prime}$ ) on $X$. Then the pair $(Y, C+H)$ (resp. $\left.\left(Y^{\prime}, C^{\prime}+H^{\prime}\right)\right)$ is a minimal model of $(X, B+D)$ (resp. $\left.\left(X, B+D^{\prime}\right)\right)$, and $Z$ (resp. $\left.Z^{\prime}\right)$ is the canonical model.

We consider a triangle $\bar{V}$ spanned by $B, B+D$ and $B+D^{\prime}$ in the space of divisors on $X$, and let $V$ be the subset corresponding to the pseudoeffective log canonical divisors. The points $(X, B+D)$ and $\left(X, B+D^{\prime}\right)$ are on the boundary of $V$. We consider a path on the boundary which connects these points, and look at the chambers whose closures intersect this path. Then the wall crossing process provides a decomposition of $\alpha$.

\section{References}

[1] Valery Alexeev, Christopher Hacon and Yujiro Kawamata. Termination of (many) 4-dimensional log flips. Invent. Math. 168 (2007), no. 2, 433-448.

[2] Urban Angehrn and Yum-Tong Siu. Effective freeness and point separation for adjoint bundles. Invent. Math. 122 (1995), no. 2, 291-308.

[3] Michael Artin. Some numerical criteria for contractability of curves on algebraic surfaces. Amer. J. Math. 84(1962), 485-496.

[4] Caucher Birkar. On existence of log minimal models. arXiv:0706.1792.

[5] Caucher Birkar, Paolo Cascini, Christopher D. Hacon and James McKernan. Existence of minimal models for varieties of log general type. math.AG/0610203.

[6] Jungkai A. Chen and Meng Chen. Explicit birational geometry of threefolds of general type. arXiv:0706.2987.

[7] Alessio Corti. Factoring birational maps of threefolds after Sarkisov. J. Algebraic Geom. 4 (1995), no. 2, 223-254.

[8] Alessio Corti et. al. Flips for 3-folds and 4-folds. Oxford Lecture Ser. Math. Appl. 35(2007), 189 pp, Oxford Univ. Press.

[9] Jean-Pierre Demailly, Thomas Peternell and Michael Schneider. Pseudo-effective line bundles on compact Kahler manifolds. Internat. J. Math. 12 (2001), no. 6, 689-741.

[10] Paolo Francia. Some remarks on minimal models. I. Compositio Math. 40 (1980), no. 3, 301-313.

[11] Osamu Fujino. Special termination and reduction theorem. in [8] http://www. math.nagoya-u.ac.jp/ fujino/index.html.

[12] Osamu Fujino and Shigefumi Mori. A canonical bundle formula. J. Differential Geom. 56 (2000), no. 1, 167-188.

[13] Christopher Hacon and James McKernan. Boundedness of pluricanonical maps of varieties of general type. Invent. Math. 166 (2006), no. 1, 1-25.

[14] Christopher Hacon and James McKernan. On the existence of flips. math.AG/ 0507597.

[15] Christopher Hacon and James McKernan. presented at conferences.

[16] Heisuke Hironaka. On the theory of birational blowing-up. Thesis, Harvard Univ., 1960. 
[17] Kazuya Kato. Logarithmic structures of Fontaine-Illusie. in Algebraic analysis, geometry, and number theory (Baltimore, MD, 1988), 191-224, Johns Hopkins Univ. Press, Baltimore, MD, 1989.

[18] Yujiro Kawamata. On the classification of noncomplete algebraic surfaces. Algebraic geometry (Proc. Summer Meeting, Univ. Copenhagen, Copenhagen, 1978), pp. 215-232, Lecture Notes in Math., 732, Springer, Berlin, 1979.

[19] Yujiro Kawamata. Characterization of abelian varieties. Compositio Math. 43 (1981), 253-276.

[20] Yujiro Kawamata. A generalization of Kodaira-Ramanujam's vanishing theorem. Math. Ann. 261 (1982), 43-46.

[21] Yujiro Kawamata. The Zariski decomposition of log-canonical divisors. in Algebraic Geometry Bowdoin 1985, Proc. Symp. Pure Math. 46 (1987), Amer. Math. Soc., 425-433.

[22] Yujiro Kawamata. On the length of an extremal rational curve. Invent. Math. 105 (1991), 609-611.

[23] Yujiro Kawamata. Termination of log-flips for algebraic 3-folds. Intl. J. Math. 3 (1992), 653-659.

[24] Yujiro Kawamata. On Fujita's freeness conjecture for 3-folds and 4-folds. alg-geom/ 9510004, Math. Ann. 308 (1997), 491-505.

[25] Yujiro Kawamata. On the cone of divisors of Calabi-Yau fiber spaces. alg-geom/ 9701006, Internat. J. Math. 8 (1997), 665-687.

[26] Yujiro Kawamata. Deformations of canonical singularities. alg-geom/9712018, J. Amer. Math. Soc. 12 (1999), 85-92.

[27] Yujiro Kawamata. On the extension problem of pluricanonical forms. math. AG/9809091, Contemporary Math. 241(1999), 193-207.

[28] Yujiro Kawamata. Flops connect minimal models. arXiv:0704.1013.

[29] Yujiro Kawamata, Katsumi Matsuda and Kenji Matsuki. Introduction to the minimal model program. in Algebraic Geometry Sendai 1985, Advanced Studies in Pure Math. 10 (1987), Kinokuniya and North-Holland, 283-360. http://faculty.ms.utokyo.ac.jp/ kawamata/index.html.

[30] Steven L. Kleiman. Toward a numerical theory of ampleness. Ann. of Math. (2) 84 (1966), 293-344.

[31] Kunihiko Kodaira. On a differential-geometric method in the theory of analytic stacks. Proc. Nat. Acad. Sci. U. S. A. 39 (1953), 1268-1273.

[32] Kunihiko Kodaira. On compact analytic surfaces. II, III. Ann. of Math. (2) 77 (1963), 563-626; ibid. 78(1963), 1-40.

[33] János Kollár et. al. Flip and abundance for algebraic threefolds. Soc. Math. France, Astérisque 211(1992).

[34] János Kollár. Effective base point freeness. Math. Ann. 296 (1993), no. 4, 595-605.

[35] János Kollár and Shigefumi Mori. Birational geometry of algebraic varieties. With the collaboration of C. H. Clemens and A. Corti. Translated from the 1998 Japanese original. Cambridge Tracts in Mathematics, 134. Cambridge University Press, Cambridge, 1998. viii+254 pp. ISBN: 0-521-63277-3.

[36] Kenji Matsuki. Introduction to the Mori program. Universitext. Springer-Verlag, New York, 2002.

[37] Yoichi Miyaoka and Shigefumi Mori. A numerical criterion for uniruledness. Ann. of Math. (2) 124 (1986), no. 1, 65-69.

[38] Shigefumi Mori. Threefolds whose canonical bundles are not numerically effective. Ann. of Math. (2) 116 (1982), no. 1, 133-176.

[39] Shigefumi Mori. Flip theorem and the existence of minimal models for 3-folds. J. Amer. Math. Soc. 1 (1988), no. 1, 117-253.

[40] Atsushi Moriwaki. Semiampleness of the numerically effective part of Zariski decomposition. J. Math. Kyoto Univ. 26 (1986), no. 3, 465-481. 
[41] David Mumford. The canonical ring of an algebraic surface. Appendix to [64]. Ann. of Math. (2) 76 (1962), 612-615.

[42] Alan Michael Nadel. Multiplier ideal sheaves and Kahler-Einstein metrics of positive scalar curvature. Ann. of Math. (2) 132 (1990), no. 3, 549-596.

[43] Noboru Nakayama. Invariance of the plurigenera of algebraic varieties under minimal model conjectures. Topology 25 (1986), no. 2, 237-251.

[44] Noboru Nakayama. Zariski-decomposition and abundance. MSJ Memoirs 14, Mathematical Society of Japan, Tokyo, 2004. xiv+277 pp. ISBN: 4-931469-31-0.

[45] Takeo Ohsawa and Kenshō Takegoshi. On the extension of $L^{2}$ holomorphic functions. Math. Z. 195 (1987), no. 2, 197-204.

[46] Gianluca Pacienza. On the uniformity of the Iitaka fibration. arXiv:0709.0310.

[47] Mihai Paun. Siu's invariance of plurigenera: a one-tower proof. J. Differential Geom. 76 (2007), no. 3, 485-493.

[48] Miles Reid. Minimal models of canonical 3-folds. Algebraic varieties and analytic varieties (Tokyo, 1981), 131-180, Adv. Stud. Pure Math. 1, North-Holland, Amsterdam, 1983.

[49] Fumio Sakai. Anti-Kodaira dimension of ruled surfaces. Sci. Rep. Saitama Univ. Ser. A 10(1982), no. 2, 1-7.

[50] V. V. Shokurov. A nonvanishing theorem. (Russian) Izv. Akad. Nauk SSSR Ser. Mat. 49 (1985), no. 3, 635-651.

[51] V. V. Shokurov. Three-dimensional log perestroikas. (Russian) Izv. Ross. Akad. Nauk Ser. Mat. 56 (1992), no. 1, 105-203; translation in Russian Acad. Sci. Izv. Math. 40 (1993), no. 1, 95-202.

[52] V. V. Shokurov. 3-fold log models. Algebraic geometry 4, J. Math. Sci. 81 (1996), no. 3, 2667-2699.

[53] V. V. Shokurov. Prelimiting flips. Tr. Mat. Inst. Steklova 240 (2003), Biratsion. Geom. Linein. Sist. Konechno Porozhdennye Algebry, 82-219; translation in Proc. Steklov Inst. Math. 240 (2003), no. 1, 75-213.

[54] V. V. Shokurov. Letters of a bi-rationalist: VII. Ordered termination. math.AG/ 0607822 .

[55] Yum-Tong Siu. Invariance of plurigenera. Invent. Math. 134 (1998), no. 3, 661-673.

[56] Yum-Tong Siu. Extension of twisted pluricanonical sections with plurisubharmonic weight and invariance of semipositively twisted plurigenera for manifolds not necessarily of general type. Complex geometry (Gottingen, 2000), 223-277, Springer, Berlin, 2002.

[57] Yum-Tong Siu. A general non-vanishing theorem and an analytic proof of the finite generation of the canonical ring. math.AG/0610740.

[58] Shigeharu Takayama. Pluricanonical systems on algebraic varieties of general type. Invent. Math. 165 (2006), no. 3, 551-587.

[59] Shigeharu Takayama. On the invariance and the lower semi-continuity of plurigenera of algebraic varieties. J. Algebraic Geom. 16 (2007), no. 1, 1-18.

[60] Hajime Tsuji. Analytic Zariski decomposition. Proc. Japan Acad. 61 (1992), 161-163.

[61] Hajime Tsuji. Pluricanonical systems of projective 3-folds of general type. math.AG/ 0204096.

[62] Eckart Viehweg. Vanishing theorems. J. Reine Angew. Math. 335 (1982), 1-8.

[63] Eckart Viehweg and De-Qi Zhang. Effective Iitaka fibrations. arXiv:0707.4287.

[64] Oscar Zariski. The theorem of Riemann-Roch for high multiples of an effective divisor on an algebraic surface. Ann. of Math. (2) 76 (1962), 560-615.

Department of Mathematical Sciences, University of Tokyo, Komaba, Meguro, TOKYo, 153-8914, JAPAN

E-mail address: kawamata@ms.u-tokyo.ac.jp 